\title{
Le pompage de l'eau par éoliennes ${ }^{(*)}$
}

\author{
PAR L. VADOT \\ INGÉNIEUR-CONSEY, AUX ÉTABLISSEMENTS NEYRPIC
}

(English text p. 524)

\begin{abstract}
Un court historique montre que, depuis la plus hante antiquité, l'énergie du vent fut utilisée pour le pompage de l'eau. Leupold et Belidor, entre autres, donnèrent des descriptions très valables de moulins pour pompage.

Les machines modernes comptent des éoliennes ientes pour pompes à piston et des éoliennes rapides dont les caractéristiques s'adaptent particulièrement bien à l'entraînement de pompes centrifuges et de pompes à hélices.

Le choix de la vitesse de démarrage, de la vitesse nominale et du point de fonctionnemenl de la pompe ont une grande influence sur la
\end{abstract}

production et l'économie du groupe de pompage. Des calculs faits, avec exemples d̀ l'appui, pour une éolienne lente entrainant une pompe à pisIon ainsi que pour une éolienne rapide anec pompe centrifuge, donnent des résultats particulièrement intéressants consignés dans des graphiques d'utilisation.

La comparaison des différents types de pompes qui peuvent ètre couplés avec une élienne rapide fait ressortir pour les hauteurs de refoulement faibles et moyennes l'avantage considérable qu'il $y$ a à utiliser une pompe d̀ pales orientables.

\section{HISTORIQUE}

A heaucoup d'autres titres de gloire, Hammourabi, fondateur de la puissance de Babylone, aurait pu ajouter celui de novateur dans le domaine de l'Hydraulique agricole. C'est Iui, assure-t-on, qui, vers l'an 2000 avant JésusChrist selon les uns, ou l'an 1700 suivant les autres, aurait concu un immense projet d'utilisation de l'énergie du vent pour assurer l'irrigation des plaines de Mésopotamie. N'y avait-il là que l'anbitieux désir d'un souverain, ou bien les audacieuses machines, comme les célèbres jardins suspendus, sont-elles à jamais retournées au limon des fleuves, nous n'en savons rien. Mais si la Perse est généralement considérée comme le berceau du moulin à vent, la Mésopotamie toute proche pourrait bien être le pays qui le premier utilisa la force du vent pour l'ćlévation de l'eau.

Sur ces antiques machines, pas de renseignements; tout au plus pouvons-nous imaginer qu'il

$\left(^{\star}\right)$ Cet article forme la suite de celui paru sous le titre: «Etude synoptique des différents types d'éoliennes », dans notre numéro 2 de 1957 , et sera suivi prochainement par un troisième article sur la production de l'énergie électrique par les éoliennes s'agissait de moulins à axe vertical assez analogues à ceux dont les vestiges subsistent encore sur les plateaux de l'Iran. La tradition veut que les moulins à vent fussent introduits en Europe par les Croisés. Sans doute est-ce un peu simplifier les choses que de faire rapporter par de glorieux chevaliers toutes les merveilles de l'Orient mystérieux; il est vrai que beaucoup de chemins menaient en Palestine, et qu'à cette époque, l'aventure commençait dès qu'était dépassé l'horizon familier. Quoi qu'il en soit, le moulin à vent paraît avoir été utilisé dans les pays méditerranéens bien avant les croisades. De là, il s'est répandu, assez lentement d'ailleurs, jusqu'au nord de l'Europe; des archives de l'abbaye de Croyland en Angleterre font état de moulins à vent dès l'an 1000. En France, d'anciens documents mentionnent la construction d'autres moulins vers 1100 . Les premiers moulins apparaissent en Hollande au xrrr ${ }^{\circ}$ siècle, en Allemagne au $x^{\circ}{ }^{\circ}$ siècle. Contrairement à leur ancêtre asiatique à axe vertical, les moulins européens sont tous à axe horizontal et, généralement, avec la roue à 4 pales en croix qui a rendu sa silhouette familière. Jusqu'au Xıv ${ }^{\circ}$ siècle, la tâche confiée à ces machines est la mou- 
DELL CARTIFICIOSE MACHINE.

Whaspar

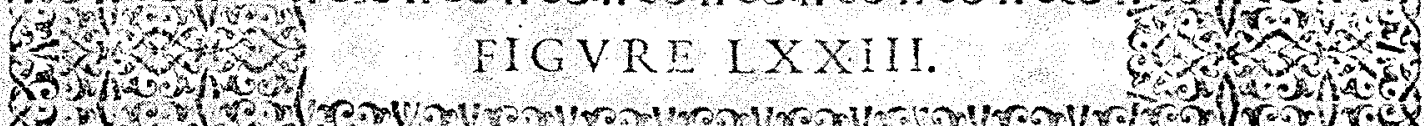
4. We 83

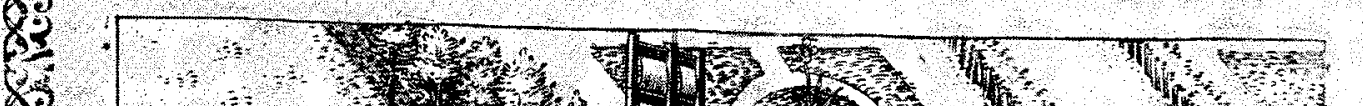

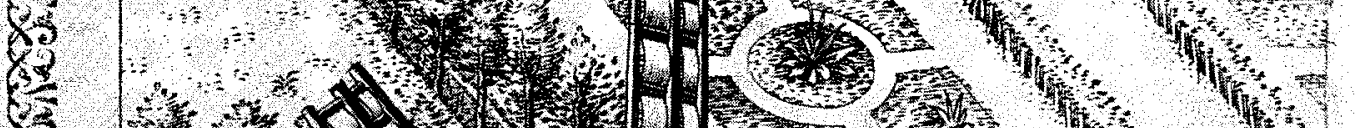

89 (n)

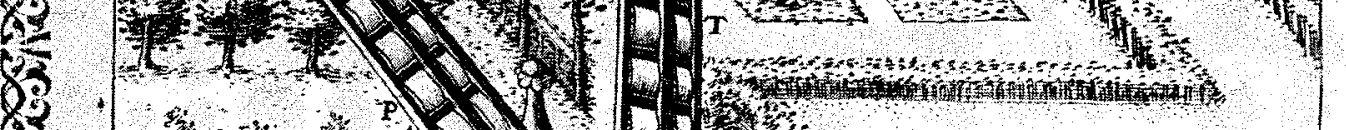

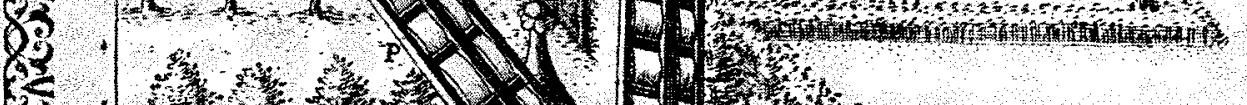

S

3

8.

\section{8.}

\section{8.}

\section{8}

\section{(8)}

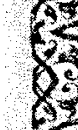

8

$x^{2}$

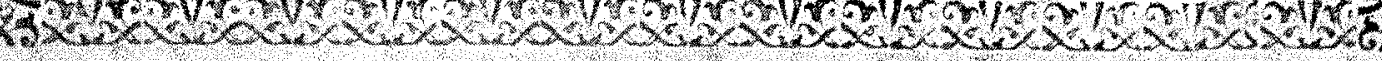

Frg. 1. - Moulin attaquant directement une double pompe à chapelet.

(Extraite de l'ouvrage "Le diverse e artificiose machine», par Agostino Ramelli, 1588.)

Windmill driving a double chain pump. 


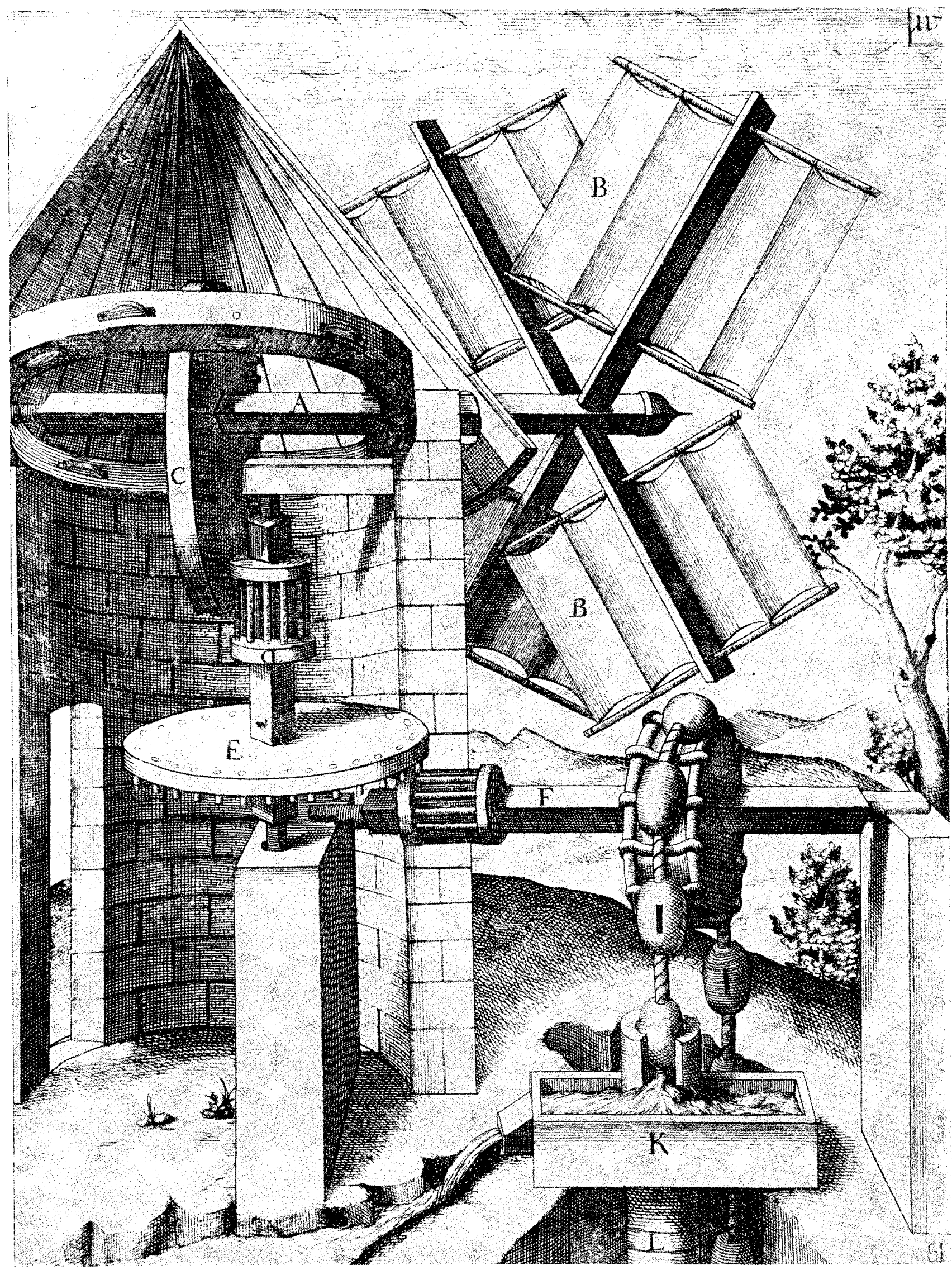

Fig. 2. - Moulin attaquant une pompe à chapelet par un double train d'engrenages; système d'orientation sur galets.

(Extraite du «Theatrum machinarum novum», d'André Böckler, 1686.)

Windmill driving a chain pump via a double gear train; yawing mounted on rollers. 


\title{
Figura Quinquagefima.
}

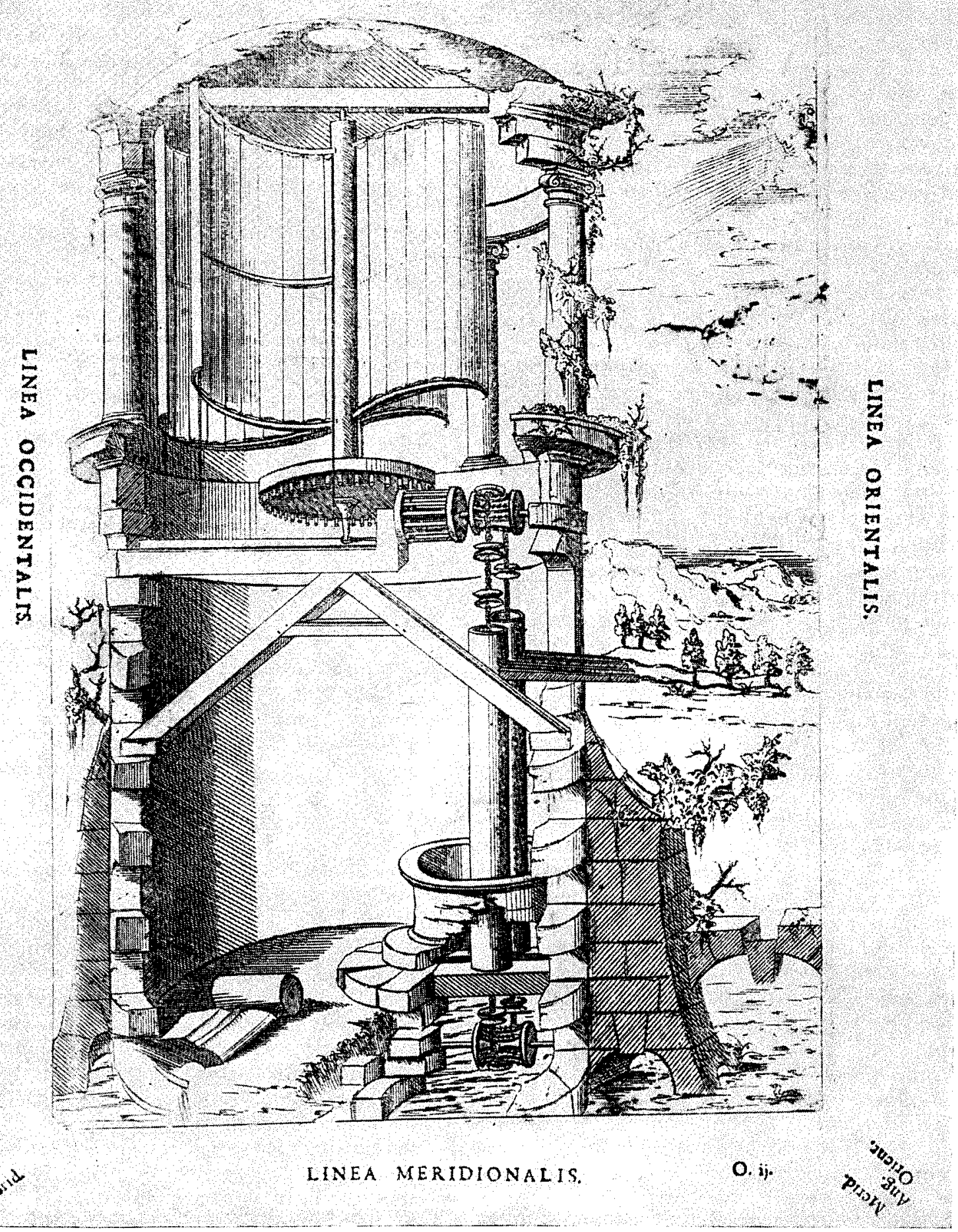

FIG. 3. - Moulin vertical ne nécessitant pas de dispositif d'orientation et commandant une pompe à chapelet. (Extraite du «Théâtre des instruments mathématiques et mécaniques»

\author{
de Jaques Besson, 1578.)
}

Vertical windmill, which does not require a yawing mechanism, driving a chain pump. 
ture du blé, d'où le nom de moulin qui leur est resté attaché.

C'est aux alentours de 1350 qu'on eut l'idée aux Pays-Bas d'employer les moulins à l'élévation de l'eau en vue d'assécher les territoires situés au-dessous du niveau de la mer. Peu à peu se créa un type de machine adapté à cette nouvelle fonction. On cite dans la région de Schoonhoven, en Hollande méridionale, plusieurs moulins de ce genre dont la construction remonterait à 1430. Les moulins d'épuisement ne tardent pas à se répandre dans un pays où ils vont devenir une nécessité vitale. De Hollande, où ils se sont perfectionnés, les moulins d'épuisement reviennent dans toute l'Europe, pompant de l'eau ici pour les besoins de l'agriculture, là pour alimenter les fontaines qui agrémentent le parc de quelque château.

On trouve des descriptions de ces appareils dans les ouvrages des ingénieurs de l'époque. Ramelli, dans son ouvrage : « Le diverse $e$ artificiose machine », offre à notre admiration un moulin de type classique actionnant une pompe à double chapelet servant à l'alimentation d'une fontaine décorative. L'ensemble du mécanisme orientable au moyen d'un cabestan, est monté sur un chemin de roulement à galets situé à la partie supérieure d'une tour servant de château d'eau.

La pompe à chapelet est fréquemment utilisée; on la rencontre dans le dessin d'une macline proposée par Böckler dans son Theatrum machinarum novum. Elle figure sur la très curieuse machine de Besson dont la roue à axe vertical rappelle les modernes rotors de Savonius. On la retrouve, enfin, dans les planches de l'Architecture Hydraulique de Bélidor.

Les figures des ouvrages des $\mathrm{xvI}^{\mathrm{e}}$ et $\mathrm{xvII^{ \circ }}$ siècles font preuve d'une imagination inspirée plus par la fantaisie décorative que par les soucis de la construction. Au xvirr siècle par contre, la technique s'impose, les mécanismes deviennent raisonnables, équilibrés, réalisables. L’ingénieur, plus que l'artiste, apparaît sous le dessin; à de nombreux détails déjà, on sent l'évolution qui conduira à des réalisations si durables qu'elles subsistent encore maintenant dans leurs grandes lignes.

La plus intéressante machine est sans conteste celle que propose Jacob Leupold dans son «Schauplatz der Wasser Künste ou Theatrum machinarum hydraulicarum imprimé en 1724 à Leipzig. Une roue à 8 aubes entraîne par un vilebrequin et une tige une pompe à piston à simple effet, suivant une disposition reprise deux siècles plus tard par les éoliennes dites américaines. Bien plus, même, la machine de Leupold est autorégulatrice. Chacune de ses aubes, pouvant pivoter autour d'un axe, est maintenue par un système de ressort tel que l'aube s'efface progressivement par grand vent, et ainsi la roue ne tourne pas plus vite lors des tempêtes que par vent moyen. Dans le même ouvrage, sont décrits quantité de mécanismes avec vilebrequin, bielle, coulisse pour la commande des pompes à piston qui tous font preuve d'une grande ingéniosité.

Bélidor, lui aussi, s'est intéressé aux machines pour élever l'eau par le moyen du vent. Dans son Architecture Hydraulique on trouve, entre autres, la description d'un moulin dont l'axe très incliné supporte d'un côté la roue éolienne, et de l'autre une roue élévatrice, sorte de tympan plongeant direclement dans le bassin d'alimentation. De semblables machines fonctionnent encore actuellement en Frise.

Bélidor donne également la description d'un moulin entraînant une noria par l'intermédiaire d'une transmission à câble constituant un réducteur, et dont les ailes affectent une forme inusitée pour l'époque, préfiguration des hélices rapides de nos jours. Il est peu probable, étant donné les moyens de l'époque et la construction rudimentaire des ailes, qu'une telle machine pût fonctionner d'une facon satisfaisante. Mais on sera, néanmoins, frappé par l'aspect moderne de l'appareil avec ses pales étroites et convenablement tordues.

Vers 1745, Edmund Lee fit breveter la roue auxiliaire « rose des vents » assurant l'orientation automatique du moulin dans le vent, dispositif excellent toujours utilisé. Cette invention serait aussi attribuée à Andrew Meilkle qui établit plus tard les ailes en persiennes.

En 1759, Smeaton présente à la « Royal Society » un mémoire : «On the construction and effects of windmill sails », mémoire où sont relatées les recherches expérimentales de l'auteur sur un dispositif de manège permettant de faire sur un modèle réduit de roues des mesures de couple à différentes vitesses. Avec Smeaton l'étude du moulin à vent entre dans une phase seientifique.

C'est certainement en Hollande que le moulin d'épuisement sous sa forme ancienne atteint le plus haut degré de perfection; il n'est pas exagéré de dire qu'une bonne partie de ce pays doit son existence à ces merveilleux appareils. Merveilleuses, certes, elles l'étaient pour l'époque, ces machines entièrement construites en bois, dont les quatre ailes en croix, atleignant près de $20 \mathrm{~m}$ d'envergure, entraînent, par un mécanisme ingénieux, des roues élévatrices, des tympans ou des vis d'Archimède. De moulin en moulin, l'eau était ainsi remontée du polder à la mer, uniquement grâce à l'énergie du vent. Aux alentours de 1630 , le lac de Schermeer, prìs d'Alkmaar en Hollande Septentrionale, fut asséché par ce procédé suivant les plans de l'ingénieur Leeghwater. Une superficie de 4.740 hec- 


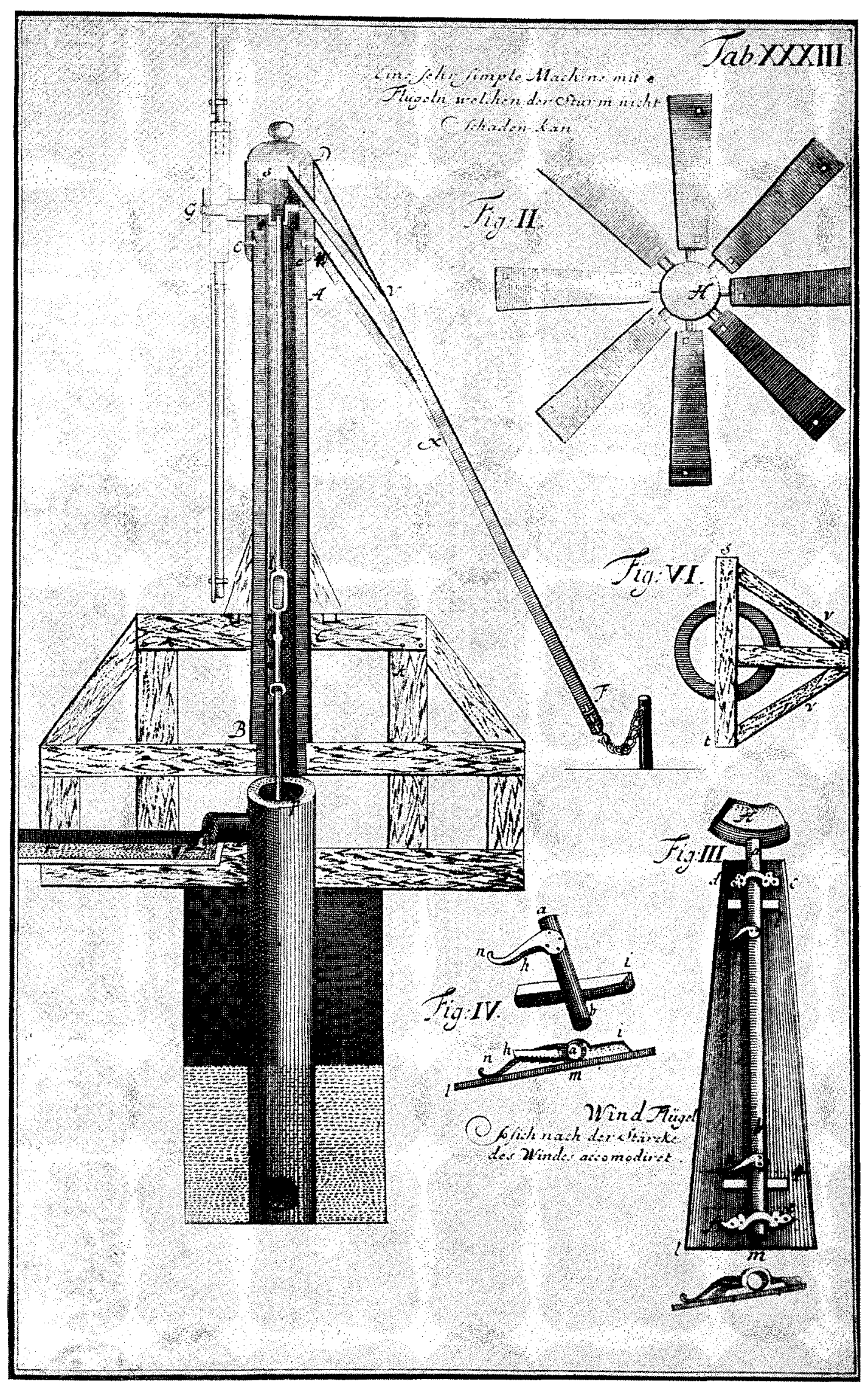

FIG. 4. - Moulin auto-régulateur à pales orientables rappelées par ressorts, commandant une pompe à piston à simple effet.

(Extraite de «Schauplatz der Wasserkünste», de Jacob Leupold, 1724.)

Self-governing windmill, with rotatable paddles having spring return, driving a single acting piston pump. 


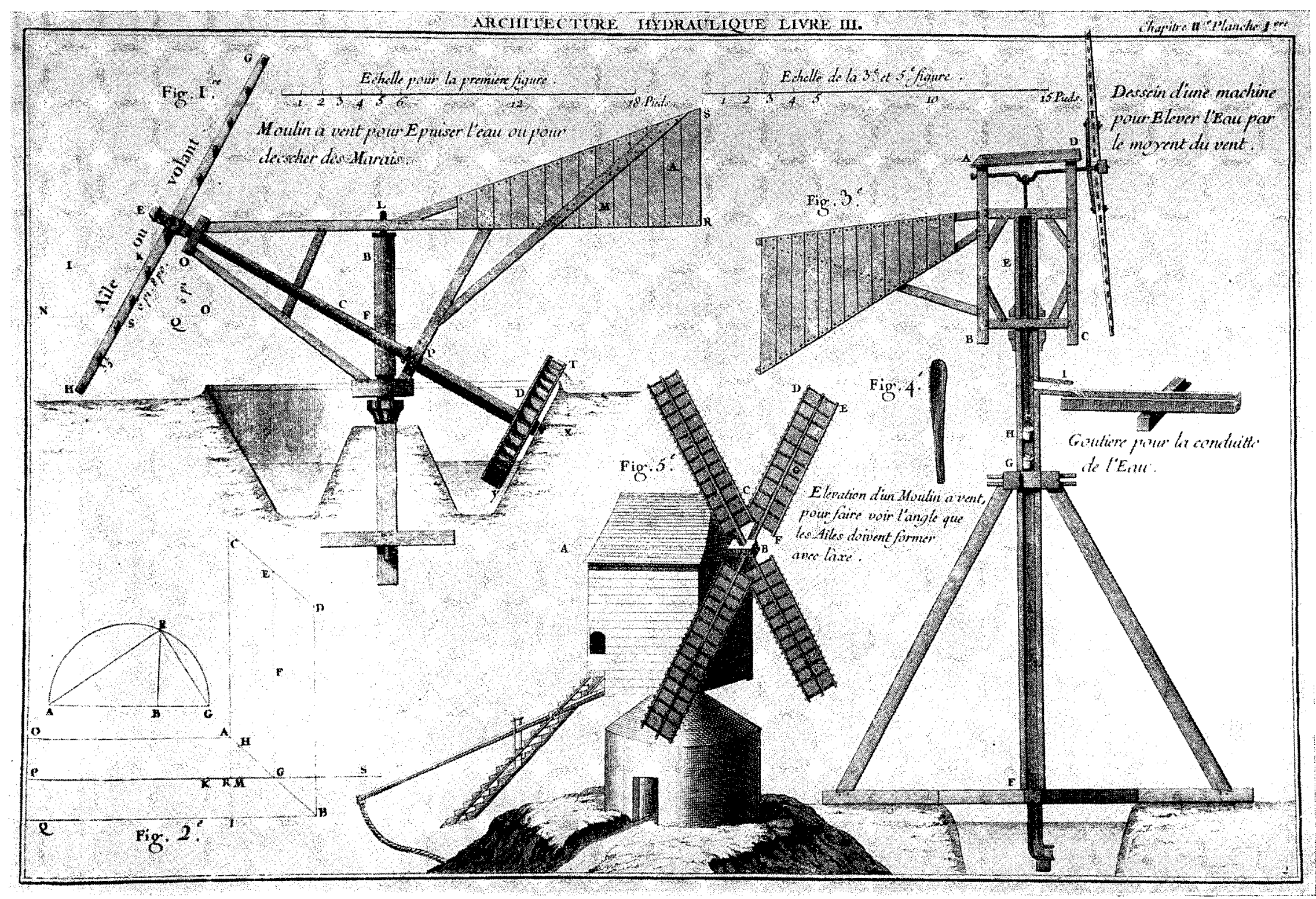

Fig. 5. - Moulin entrainant directement un tympan, et moulin actionnant une pompe à piston. (Extraite de l' «Architecture hydraulique», de Belidor, 1739.)

Windmill driving a treadmill directly and a windmill a piston pump. 


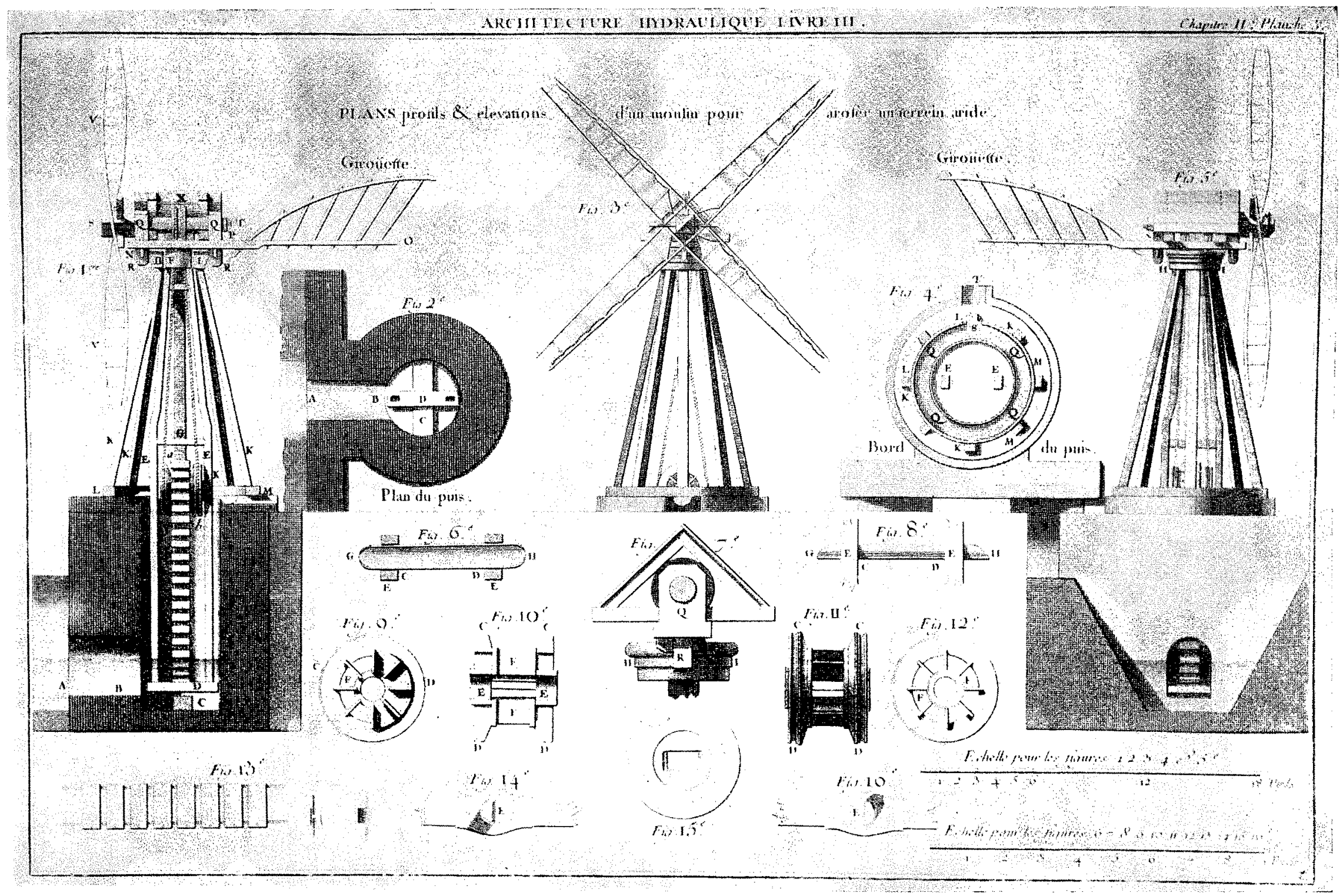

Fic. 6. - Moulin entrainant une pompe à chapelet par une transmission à câble,

(Extraite de l" «Architecture hydraulique», de Belidor, 1739.)

Windmill driving a chain pump through a cable transmission. 
tares fut récupérée et drainée par un ensemble de 50 moulins refoulant l'eau en deux étages. Le même Leeghwater avait prévu l'asséchement de la mer de Haarlem au moyen de 160 moulins à vent. Voilà bien des entreprises à l'échelle de notre vingtième siècle! N'en peut-on pas conclure que le moulin à vent de pompage avait atteint, dès le xvII ${ }^{\circ}$ siècle, un développement quasi industriel?

Le très grand nombre de machines utilisées par les Hollandais les a conduits à une véritable standardisation, en sorte que, dans chaque région, les moulins se ramènent à quelques types seulement, répétés à un grand nombre d'exemplaires : Wipmolen perché sur sa base pyramidale, Weidemolen dont la tour octogonale sert d'habitation, Binnenkruier aux formes trapues. Après des siècles de bons et loyaux services, le moulin reste une des caractéristiques du paysage hollandais.

\section{Données météorologiques}

Avant toute chose, la connaissance de l'intensilé des vents au lieu d'utilisation est nécessaire à l'établissement d'un projet d'équipement de pompage par éolienne. Durant une période suffisamment longue d'au moins une année, des mesures, ou mieux, des enregistrements de l'intensité du vent devront être faits au point considéré. Au cas où, pour des raisons d'économie, il ne serait pas possible d'effectuer l'enregistrement d'une année complète, il convient d'obtenir des enregistrements typiques des différentes périodes de l'année, pour tenir compte de l'influence saisonnière sur le régime des vents. L'appareil de mesures sera placé autant (que possible sur un support dont la hauteur sera voisine de celle de l'colienne prévue. Les relevés météorologiques se font sourent à la hauteur standard de $10 \mathrm{~m}$, mais généralement l'axe des roues sera à une hauteur supérieure, de l'ordre de $15 \mathrm{~m}$. Il serait préférable d'adopter pour l'appareil de mesure une hauteur de $15 \mathrm{~m}$, ce qui évitera la transposition des résultats de la hauteur $10 \mathrm{~m}$ à la hauteur $15 \mathrm{~m}$, transposition assez hasardeuse, dans l'ignorance où l'on est généralement de la loi locale de variation de l'intensité du vent avec l'altitude. Certes, on admet généralement que la vitesse du vent varie comme $\mathrm{H}^{1 / 5}$, mais ce n'est là qu'une Ioi approximative dont on peut s'écarter notablement suivant la rugosité moyenne de la région environnante. En outre, cette loi n'est guère valable sí on opère dans la couche directement perturbée par les obstacles voisins, ce qui sera très souvent le cas. Il est à peine besoin de préciser que les enregistrements effectués à trop petite dis- tance du sol ou au voisinage d'obstacles tels que bâtiments, arbres, etc., n'ont aucun sens.

On utilise parfois comme appareils de mesure des appareils du type compteur totalisateur d'énergie domnant directement l'énergie théoriquement disponible par mètre carré de surface offerte au vent pendant la période qui sépare deux lectures consécutives. Ces appareils ont l'avantage d'être d'une exploitation économique; ils ne donnent toutefois que des renseignements sur l'importance de l'énergie éolienne disponible; ils sont par contre incapables de donner les grandeurs fondamentales nécessaires à la détermination d'un équipement. Aussi les compteurs d'énergie éolienne ne sont-ils à utiliser que pour une prospection grossière d'une région. L'anémomètre ordinaire à lecture directe est pratiquement sans valeur, par suite des grandes variations d'intensité du vent. Finalement le seul appareil à conseiller est l'anémomètre enregistreur donnant en fonction du temps les variations de l'intensité du vent. Tous les renseignements nécessaires peuvent être tirés de ces enregistrements. Il convient, toutefois, pour la facilité de l'interprétation, de traduire les enregistrements en un langage simple se ramenant à la détermination des éléments caractéristiques suivants: courbe de vents classés ou courbe vitesse-durée, courbe de fréquence des vents ou des énergies, courbe des débits pompés, etc.

La courbe des vents classés ou courbe de vitesse-durée indique pour une période donnée, et pour chaque valeur de la vitesse du vent, le nombre d'heures pendant lequel la vitesse du vent est supérieure ou égale à la vitesse consi-

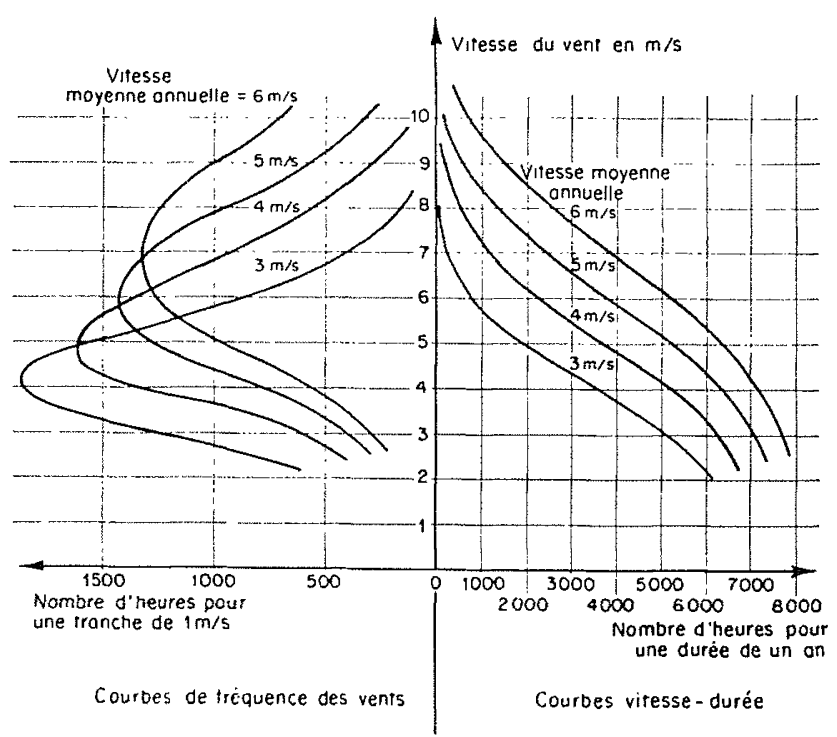

FIG. 7. - Courbes de fréquence des vents et de vitessedurée pour différentes valeurs de la vitesse moyenne annuelle.

Wind frequency and Velocity-duration curves for different values of annual mean wind speed. 
dérée. Cette courbe est généralement établie pour une année, mais, dans les régions à régime saisonnier très marqué, il est plus indiqué de l'établir saison par saison. La détermination est faite à partir de l'enregistrement de l'anémomètre; le dépouillement correspondant est assez fastidieux; il peut être grandement facilité par l'emploi d'un appareil lecteur spécial.

Les courbes vitesse-durée présentent à peu près toutes la même allure. Notons que la zone relative aux faibles vitesses de vent est généralement sujette à caution, par suite de l'insensibilité des anémomètres, disons au-dessous de $2 \mathrm{~m} / \mathrm{s}$; mais cette zone est de peu d'importance au point de vue énergétique. On peut, en gros, caractériser les courbes vitesse-durée par la valeur moyenne du vent pour la période considérée, vent appelé 《 kent moyen annuel ». Ce chiffre moyen ne donne évidemment aucune indication sur la régularité du vent.

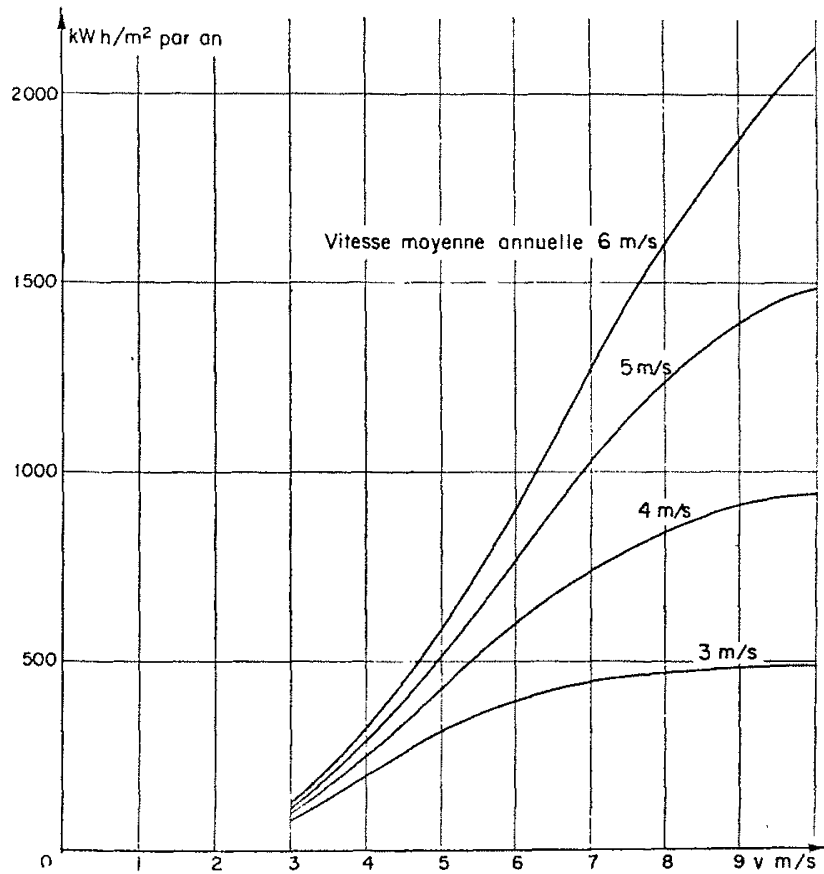

FIi. 8. - Energie totale disponible dans l'année par mètre carré de surface balayée, en fonction de la vitesse de vent nominale, pour différentes valeurs de la vitesse moyenne annuelle (pour obtenir l'énergie effectivement récupérable, il faut multiplier ces chiffres par le coefficient de puissance de l'appareil).

Total available power in one year per square metre of swept area in terms of the rated wind speed, for various values of the annual mean wind speed (To obtain the effectively recoverable power, these figures should be multiplied by the power coefficient of the equipment).

La courbe de fréquence des vents, par contre, donne une idée de la régularité des vents; cette courbe, qui se déduit très simplement de la courbe vitesse-durée, indique le nombre d'heures, pour la période considérée, pendant lesquelles la vitesse du vent reste comprise dans une tran- che donnée; elle représente done simplement les variations de l'inverse de la pente de la courbe précédente. Cette courbe présente toujours un maximum plus ou moins prononcé. Généralement, la vitesse du vent correspondant au maximum de la courbe de fréquence croìt en même temps que la vitesse moyenne pour la période considérée. Il peut arriver, dans les pays à régime particulier, que la courbe de fréquence présente deux maximums.

La courbe de fréquence, qui donne des renseignements sur le régime des vents sera utilement complétée par la courbe des énergies disponibles. La puissance fournie par le vent étant proporlionnelle au cube de la vitesse, on obliendra une courbe donnant, pour chaque tranche de vent, une quantité proportionnelle à l'énergie disponible en multipliant les ordonnées des courbes de fréquence de vent par le cube de la vitesse. On obtient ainsi encore des courbes présentant un maximum, mais, alors que pour les fréquences de vent, la valcur absolue du maximum diminue quand le vent moyen augmente, par contre, l'énergie correspondant au maximum croit lorsque le vent moyen augmente.

Afin de faciliter la détermination de la puis-

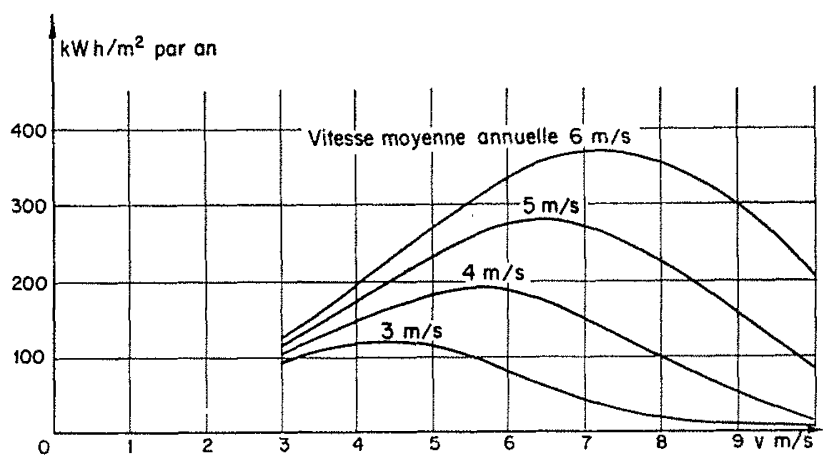

Fra. 9. - Energie supplémentaire disponible dans l'année par mètre carré de surface balayée, pour un accroissement de la vitesse de vent nominale de $1 \mathrm{~m} / \mathrm{s}$, en fonction de la vitesse du vent et pour différentes valeurs de la vitesse moyenne annuelle (pour obtenir l'énergie effectivement récupérable, il faut multiplier ces ehiffres par le coefficient de puissance de l'appareil).

Additional power avalable in one year per square metre of swept area, for an increase of 1 metre/second in the rated wind speed, in terms of the wind speed and taking various values for the annual mean wind speed (To obtain the effectively recoverable power, these figures should be multiplied by the power coefficient of the equipmen().

sance à équiper, il sera utile de tracer les courbes donnant l'énergie produite par tranche de vent, c'est-à-dire l'accroissement d'énergie récupérable lorsque la vitesse de vent, pour laquelle sera prévue la machine, passera de $v \dot{\mathrm{a}} v+\Delta v$. Toutes ces courbes se déduisent sans peine de la courbe vitesse-durée résumant les réenregistrements. 
Lorsque l'énergie produite par l'éolienne ne peut pas être simplement utilisée au fil du vent, comme ce serait le cas pour une machine débitant de l'énergie électrique sur un réseau de grande capacité, il faut faire entrer en ligne de compte les possibilités d'accumulation de cette énergie, éminemment variable avec le temps, pour l'adapter à une utilisation régulière. Le problème de l'accumulation se pose pour les éoliennes de production d'énergie électrique débitant sur un réseau indépendant; il se pose également pour les éoliennes de pompage destinées à alimenter un poste d'eau indépendant de tout réseau d'alimentation, ce qui sera très fréquemment le cas. Le même problème se présente enfin pour les éoliennes de drainage, mais dans ce cas le réservoir d'alimentation se trouve à l'aspiration au lieu d'être au refoulement.

La résolution du problème de l'accumulation dans un réservoir conduit à déterminer l'évolution dans le temps du volume d'eau poinpé par la machine et à la comparer aux besoins en eau. Dans le cas simple où le débit demandé est constant, l'équipement optimum, du point de vue énergétique, serait tel que le débit moyen

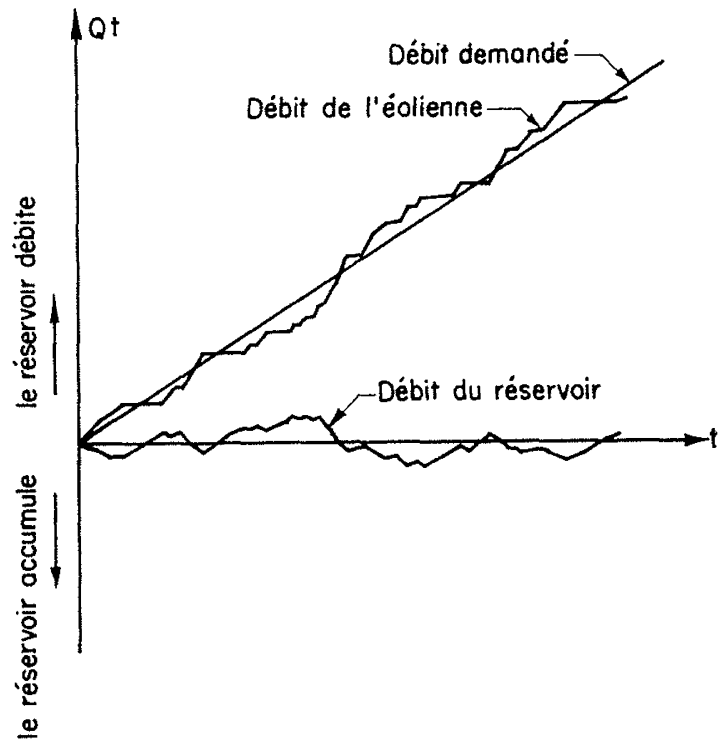

Frg. 10. - Variation, dans le temps, des volumes d'eau, pompé par l'éolienne et demandé, pour la détermination $\mathrm{du}$ volume du réservoir d'accumulation.

Variation, with time, of the water volumes pumped by the windmill and demanded, for the determination of the storage reservoir volume.

pompé dans une période donnée, d'une année par exemple, par l'éolienne, soit égal au débit demandé. Par suite des irrégularités de pornpage, il faudra prévoir un réservoir dont la capacité dépendra du degré d'irrégularité des vents. On peut facilement représenter les variations de débit pompé dans un graphique débit cumulé-temps; on obtient ainsi une courbe sans cesse croissante, plus ou moins tourmentée. Dans un tel graphique, un débit demandé constant sera représenté par une droite passant par l'origine. La différence entre les ordonnées de la courbe de l'éolienne et de la droite de débit consommé représentera la part du réservoir d'accumulation. On obtient ainsi la courbe représentant l'évolution dans le temps. du débit fourni ou absorbé par le réservoir $Q_{r}$. L'aire située sous cette courbe représente les volumes d'eau totaux fournis ou absorbés :

$$
\mathrm{V}=\int_{0}^{t} \mathrm{Q}_{r} d t
$$

Si l'éolienne est bien adaptée au débit demandé, la courbe du volume du réservoir oscillera plus ou moins régulièrement autour de la valeur nulle. Si l'éolienne est trop forte pour la demande, la courbe du réservoir serait décroissante, ce qui signifie que le débit d'apport est plus grand que le débit demandé; si au contraire l'éolienne était insuffisante, la courbe du volume du réservoir serait toujours croissante, aux irrégularitẻs près, évidemment.

L'éolienne étant convenablement choisie, la capacité du réservoir sera définie par le maximum de la courbe :

$$
\mathrm{V}=\int_{0}^{t} \mathrm{Q}_{r} \mathrm{dt}
$$

Il est bien évident que dans la pratique il conviendra d'adapter la consommation aux irrégularités saisonnières de production, mais les irrégularités journalières ou de quelq̣ues jours devront être absorbées par le réservoir. Il convient donc d'établir la courbe représentative de l'évolution dans le temps du débit pompé; cette courbe sera établie à partir des enregistrements anémométriques en utilisant une courbe donnant, en fonction de la vitesse du vent, le débit pompé pour une machine donnée. Cette détermination est fastidieuse; elle sera grandement facilitée par l'emploi d'un apparcil automatique de dépouillement, appareil permettant d'ailleurs d'obtenir les résultats pour plusicurs types de machines, à partir d'un même enregistrement.

\section{Appareils de dépouillement des enregistrements anémométriques}

Pour rendre utilisables les enregistrements anémométriques, il est nécessaire de les traduire en courbes vitesse-durée ou fréquence-vitesse. Ce travail de dépouillement peut être fait mécaniquement au moyen d'un appareil spécial. Cet appareil comporte un dispositif de déroulement du papier enregistrcur situé en facc d'un ensem- 
ble de contacts électriques échelonnés suivant l'échelle des vitesses. Un frotteur coulissant peut se déplacer sur ces contacts, un stylet relié à ce frotteur permet, en suivant la courbe à la main, au fur et à mesure de son avance, d'amener le frotteur en présence du contact correspondant à la vitesse indiquée par la courbe. Chaque contact ferme le circuit d'un compteur d'impulsions alimenté par un générateur d'impulsions, constitué simplement par une batterie et une roue dentée formant rupteur, et dont la vitesse de rotation cst proportionnelle à la vitesse de déplacemenl du papier.

Dans ces conditions, tous les compteurs élémentaires correspondant à une vitesse égale ou inférieure à celle indiquée par le stylet suiveur, avancent d'un nombre d'unilés correspondant au chemin parcouru sur le papier. En cours de dépouillement, ii suffit de relever les chiffres donnés par les compteurs pour obtenir, à un instant quelconque, l'état de la courbe vitessedurée. Il est également possible de compléter cet appareil par un dispositif permettant d'obtenir la courbe des débits pompés pour une éolienne donnée. Dans ce but, une deuxième série de conlacts est placée à còté de la précédente. Chacun de ces contacts est relié à une résistance dont la valeur est inversement proportionnelle au débit fourni par l'éolienne pour la vitesse considérée. Le contact coulissant relié au stylet suiveur ferme un circuit comprenant une source de courant et un compteur. La vitesse de déroulement étant constante, le compteur indique à chaque instant le volume d'eau pompé. On peut naturellement monter sur la même série de contacts un autre ensemble de résistances alimentant un autre compteur, et représentant une autre machine; il est ainsi possible au cours d'un même dépouillement, d'obtenir les débits pompés par des éoliennes de caractéristiques différentes.

\section{Utilisation des données météorologiques pour la détermination des caractéristiques de l'installation}

Etant en possession des différents éléments météorologiques, voyons maintenant comment les utiliser pour définir les caractéristiques d'un équipement. Le fonctionnement de l'éolienne est lié à trois valeurs de la vitesse du vent :

$1^{\circ}$ la vitesse $v_{1}$, dite 《vitesse d'amorçage », est la vitesse pour laquelle la machine commence à fournir de l'énergie, c'est-à-dire, dans le cas d'une installation de pompage, la vitesse pour lacjuelle la pompe commence à débiter. Cette vitesse ne doit pas être confonduc avec la « vitesse de démarrage $\gg v_{0}$ pour laquelle la machine commence à tourner, mais sans fournir d'énergie utilisable. Dans le cas de l'entraînement d'une pompe à piston, $v_{0}$ et $v_{1}$ sont confondues, mais dans le cas de l'entraînement d'une pompe centrifuge, $v_{0}$ et $v_{1}$ sont notablement différentes.

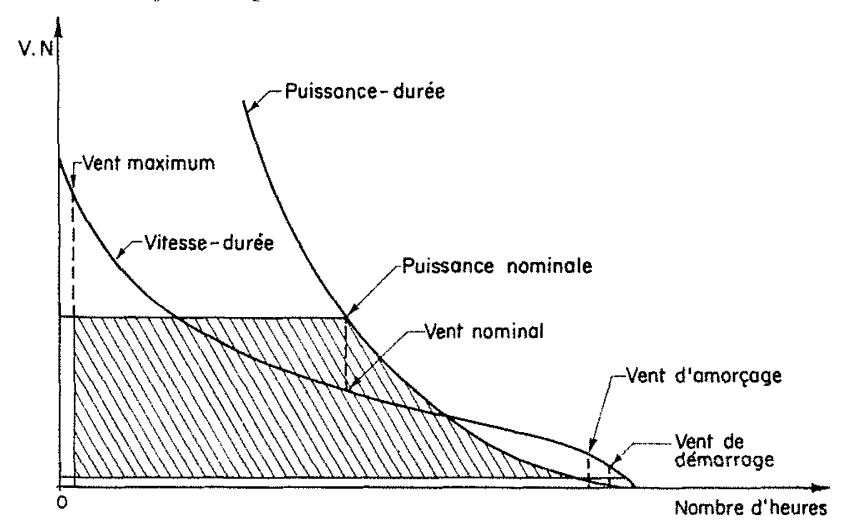

Fug. 11. Conditions d'emploi d'une éolienne. Itilization conditions of a windmill.

$2^{\circ}$ la vitesse $v_{n}$, dite « vitesse nominale », est la vitesse pour laquelle la pleine puissance de l'équipement est atteinte. A partir de cette vitesse, le dispositif de réglage intervient pour limiter la puissance fournie par la machine et la maintenir constante.

$3^{\circ}$ la vitesse $v_{\operatorname{mix}}$, dite « vitesse de mise hors service », est la vitesse à partir de laquelle la machine est éventuellement mise hors service sous l'influence du réglage ou d'un dispositif de sécurité.

Ces trois vitesses définissent, sur le diagramme de vitesse-durée ou énergie-durée, la zone d'utilisation. Les déterminations des vitesses $v_{1}$ et $v_{n}$ sont de la plus grande importance. La vitesse $v_{\text {max }}$ dépend uniquement du mode de réglage de l'appareil, elle est sans grande influence sur l'énergie produite, car la durée des vents violents est faible. Si on se place uniquement au point de vue de l'énergie totale produite, le choix de la vitesse $v_{1}$ de démarrage est sans grande importance, car l'énergie produite par vent faible est toujours très réduite. Par contre, dans le cas d'une machine fonctionnant isolément et comportant un réservoir d'accumulation, le choix de cette vitesse est très important, car c'est elle qui, en définitive, détermine la grandeur du réservoir à prévoir.

\section{Détermination de la vitesse d'amorçage}

Il est évident qu'il y a intérêt à réduire autant que possible la valeur de cetle vilesse afin d'aug- 
menter le nombre d'heures de fonctionnement de l'installation. Toutefois, la détermination de cette vitesse résulte d'un compromis entre le prix du réservoir et celui de la machine. En effet, si l'importance du réservoir diminue lorsque la vitesse de démarrage baisse, par contre, le diamètre de l'éolienne augmente lorsque cette vitesse diminue, puisque, pour une installation donnée, le couple absorbé par la pompe à l'amorçage reste constant. Le problème de la détermi-

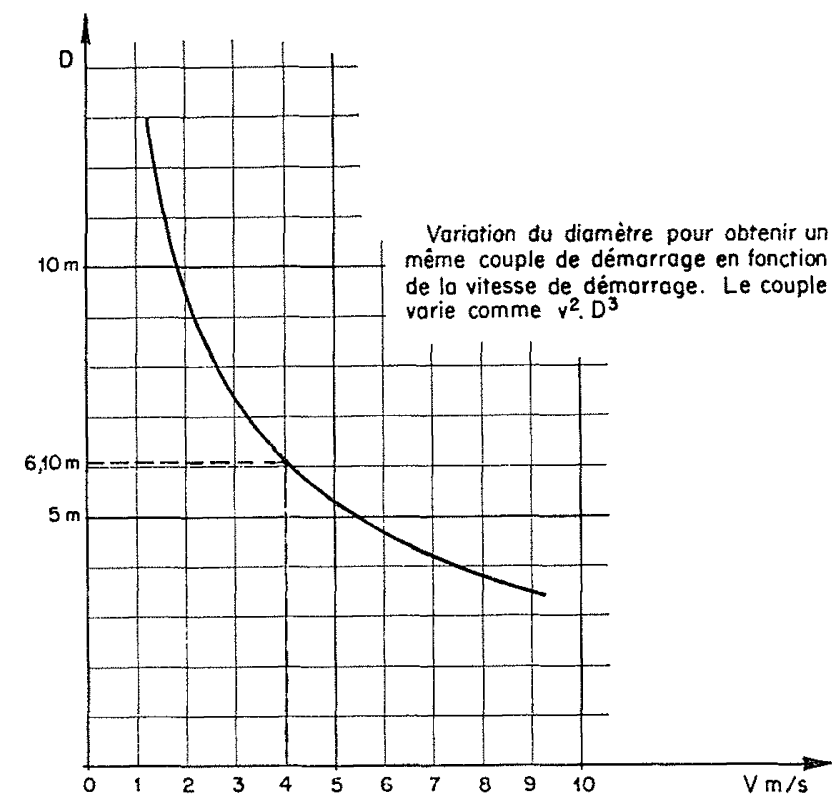

Fra. 12. -- Variation du diamètre d'une éolienne lente avec la vitesse de démarrage, pour en obtenix un même couple de démarrage, en vue de la commande d'une pompe à piston.

Variation of the diameter of a slow-running windmill against the starting wind speed, to obtain the same starting torque, when considering the drive to a piston pump.

nation de la vitesse de démarrage se pose de façon différente suivanl que l'éclienne entraîne une pompe volumétrique ou une pompe du genre centrifuge.

Dans Je premier cas, il s'agit d'un problème de couple de démarrage; on ne peut agir que sur le diamètre de l'colienne ou sur le rapport de démultiplication du mécanisme, car la pompe est du type à couple constant.

Dans le deuxième cas, par contre, les choses sont plus complexes, car non seulement la dimension et la vitesse de l'éolienne sont à déterminer, mais encore le point de fonctionnement de la pompe peut varier suivant le diamètre de la roue de la pompe, et sa vitesse de rotation. II ne s'agit plus simplement d'obtenir un certain couple à vitesse nulle, comme pour la pompe à piston, mais d'obtenir un couple à une certaine vitesse, ces deux grandeurs dépendant des caractéristiques de la pompe centrifuge.

\section{Détermination de la vitesse nominale}

Le choix de cette vitesse est principalemenl lié à la production totale d'énergie dans l'année. Deux points de départ peuvent être envisagés : soit que les caractéristiques de la pompe soient imposées: hauteur, débit, soit que le diamètre et le type de l'éolienne soient imposés. Si les rendements de l'éolienne et de la pompe restaient constants aux charges fractionnaires, il $\mathrm{y}$ aurait intérêt à choisir pour $v_{n}$ une valeur élevée. Mais ce n'est généralement pas le cas; compte tenu des variations de rendement de l'éolienne et de la pompe, on cherchera à obtenir le maximum de volume d'eau dans l'annće pour une éolienne donnée, ou au contraire à obtenir l'éolienne la plus petite pour un volume d'eau donné.

Dans le cas d'un entrainement de pompe centrifuge, le choix de la vitesse nominale ne peut être fait indépendamment du choix de la vitesse d'amorcage, le rapport de ces vitesses étant imposé pour une pompe donnée. En effet, sous une hauteur donnće, l'écart de vitesse de rotation de la pompe entre la marche à rendement maximum et la marche à débit nul ou à l'amorçage est faible. On ne peut accroître cet écart qu'en choisissant un point de fonctionnement s'écartant notablement du rendement maximum. Il faudra donc, pour une pompe donnée, chercher à déplacer l'ensemble des points $v_{1}$ et $v_{n}$ sur la courbe de vitesse-durée, de facon à réaliser le meilleur compromis entre volume d'eau total pompé et volume du réservoir nécessaire pour tenir compte des périodes de calme, ou bien choisir une pompe telle qu'elle permette de réaliser un rapport convenable entre $v_{1}$ et $v_{n}$; on ne peut toutefois adopter pour ce rapport une valeur trop forte sans s'écarter des zones de rendement acceptable. La difficulté qu'il y a à obtenir une valeur convenable pour le rapport $v_{n}$ sur $v_{1}$ pourrait être tournée dans une certaine mesure en faisant varier le rapport de multiplication entre colienne et pompe. Mais on serait conduit à des solutions coûteuses, car la variation du rapport de multiplication devrait être automatique pour pouvoir jouer à chaque saute de vent; il ne semble pas qu'une telle solution ait été industriellement réalisée jusqu'à maintenant.

\section{Matériel normalement utilisé}

Deux types de groupe de pompage s'offrent à l'utilisateur : les éoliennes lentes avec pompe à piston, existant actuellement en très grand nombre, et les éoliennes rapides accouplées à des pompes à hélice ou à des pompes centrifuges; 
ces dernières machines, de réalisation récente, caractérisent l'évolution des techniques nouvelles.

a) Installation DE PETite PUissance AVEC POMPE A PISTON :

$\mathrm{Au}$ premier abord, il ne paraît pas évident, et en toute rigueur il n'est pas obligatoire, qu'une installation de pompage de petite puissance soit équipée avec une pompe à piston. Mais, d'une part, l'importance des hauteurs de refoulement, d'autre part, la nécessité d'utiliser un matériel simple, conduisent généralement, pour les petites puissances, à l'emploi de pompes à piston lentes.

L'éolienne à utiliser dans ce cas sera une machine à faible vitesse spécifique avec presque toujours un démultiplicateur. Ce genre de machine possède un couple de démarrage élevé qui s'accorde parfaitement avec les exigences des pompes à piston. Les roues à faible vitesse spécifique comportant un grand nombre de pales, on ne peut envisager que l'emploi de pales fixes;

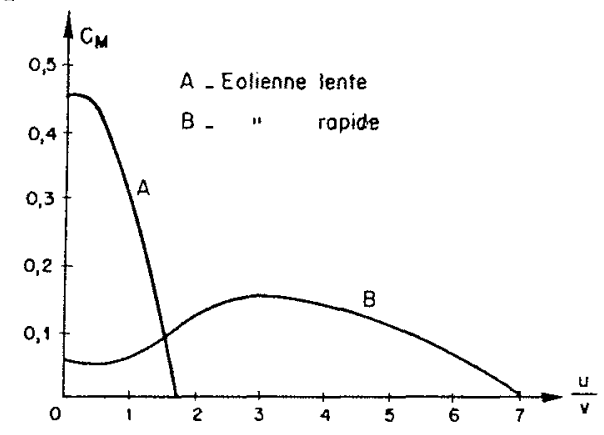

lig. 13. - Coefficient de couple des éoliennes lente et rapide, en fonction de la vitesse périphérique.

Torque coefficient for high speed and slow-running windmills, in terms of the tip speed ratio.

le mode de réglage qui s'impose alors pour la limitation de la vitesse, de la puissance et des efforts au grand vent est le réglage par éclipsage. Le genre d'appareil auquel on est ainsi conduit ne peut être raisonnablement construit au-delà d'une certaine dimension. Par suite de l'inertie des roues à grand nombre de pales, le comportement d'une telle machine dans les rafales en interdit l'emploi pour les grands diamètres. Pour fixer les idées, un diamètre de 7 à $8 \mathrm{~m}$ est un maximum.

D'autre part, la pompe à piston étant très sensiblement une pompe à couple constant, la puissance de' l'éolienne est mal utilisée au grand vent. Pour de petites machines ce dernier point n'est pas grave, mais, pour de grosses machines où la notion de rendement a plus d'importance, cette mauvaise utilisation serait inacceptable. On a essayé d'y remédier par des dispositifs modifiant la course de la pompe en fonction de la vitesse du vent, mais rien de pratiquement utilisable n'est à retenir dans ce sens.

b) Installation DE moyenNe ou grande puisSANCE AVEG POMPE ROTATIVE :

Les inconvénients qui viennent d'être signalés condamnent l'emploi des pompes à piston pour le pompage de grande puissance, sauf, bien entendu, si la hauteur de refoulement l'exige, et alors des dispositions particulieres s'imposent. On est alors conduit, suivant les débits et les hauteurs, à utiliser des pompes centrifuges ou des pompes à hélice. Ces pompes doivent tourner vite, d'où l'emploi d'éoliennes à grande vitesse spécifique, généralement avec multiplicateur. Le faible couple de démarrage des machines n'est pas un inconvénient grave, puisque le couple de démarrage des pompes rotatives est également faible. Naturellement, le réglage de la vitesse ne s'impose pas; seul doit être résolu le problème du comportement au grand vent. De tels groupes permettent une bonne utilisation de l'énergie éolienne; ils ont toutefois l'inconvénient de ne fournir qu'une faible hauteur de refoulement au démarrage. Si les vents faibles n'entrent que pour une faible part dans le bilan énergétique annuel, cet inconvénient est sans gravité. Ces groupes sont donc bien adaptés aux régions présentant des vents assez constants.

Dans le cas de faible hauteur de refoulement, on emploierait avantageusement une combinaison de l'éolienne rapide avec une pompe-hélice à pales automatiquement orientables, l'inclinaison des pales diminuant par vent faible pour réduire la puissance absorbée. Une telle disposition permettrait d'allier l'amorçage par vent faible avec l'obtention de grand débit par vent fort, le rendement du groupe restant toujours voisin du maximum; cette solution est évidemment un peu compliquée, du point de vue mécanique, mais elle doit être considérée sérieusement pour des groupes d'une certaine puissance à faible hauteur de refoulement.

Pour les grosses puissances, enfin, la transmission électrique entre l'éolienne et la pompe peut s'imposer; il ne faut pas perdre de vue, en effet, quei les zones les plus favorables pour l'énergie éolienne sont rarement celles où se trouvent les sources ou les puits. La transmission électrique a le gros avantage de supprimer toutes les difficultés de liaison mécanique, qui entraînent de grosses dépenses pour les grandes puissances; elle a en outre l'avantage de permettre, éventuellement, l'emploi direct de l'énergie électrique.

\section{Eoliennes lentes pour pompe à piston}

Ces machines comportent une roue à grand nombre (de 16 à 24) d'aubes fixes réalisées en 
tôle et non profilées. Cette roue atlaque par l'intermédiaire d'un réducteur à engrenages un mécanisme de transformation du mouvement de rotation en mouvement de translation : bielles, coulisses, excentriques, etc. Tous ces méca-

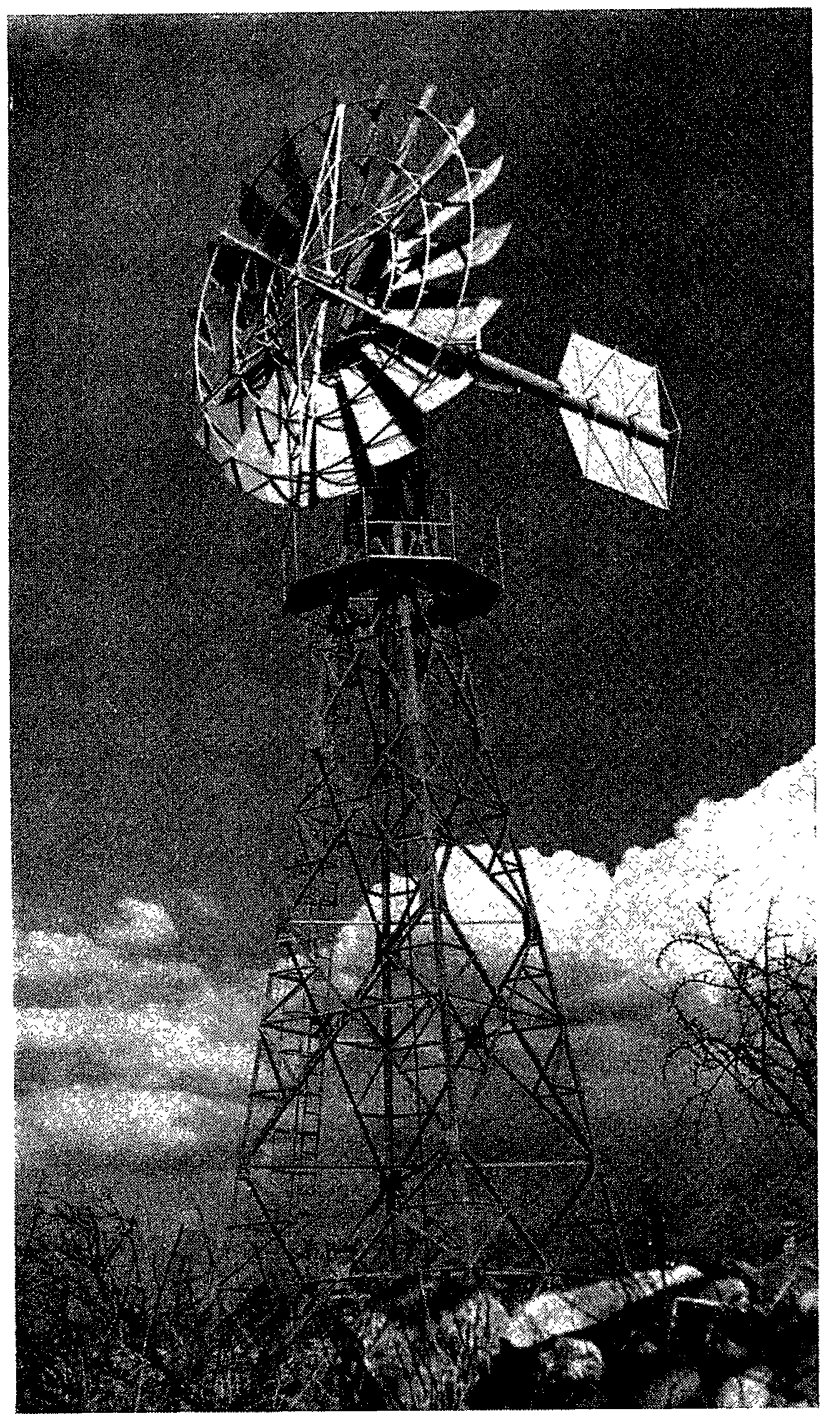

FIG. 14. - Eolienne lente pour pompe à piston avec roue de $6,10 \mathrm{~m}$ de diamètre. Construction Neyrpic.

Slow-running windmill for reciprocating pump. Wheel diameter: $6.1 \mathrm{~m}$. Manufactured by Neyrpic.

nismes sont à peu près équivalents, le rendement dépendant plus de la qualité de l'exécution que du principe utilisé. La réduction de vitesse entre la roue et le mécanisme de commande de la pompe s'impose pour les grandes profondeurs d'épuisement, pour réduire d'une part, les efforts d'inertie et éviter, d'autre part, les phénomènes de résonance dans la tige de manœuvre qui, par suite de sa longueur, se comporte comme un ressort de traction. La construction en série s'imposant pour ce matériel, le réducteur de vitesse est conservé même pour les faibles profondeurs d'épuisement. Généralement, un même mécanisme permet d'obtenir deux valeurs de la course de la pompe, par simple démontage du maneton. Le réglage de la puissance au grand vent s'obtient toujours par éclipsage de la roue, celle-ci se repliant dans le lit du vent en cas de tempête. Dans ce but, l'empennage est articulé et lié au corps de l'appareil par un ressort; le moment d'éclipsage s'obtient soit par une palette latérale, soit, beaucoup plus fréquemment, en excentrant légèrement l'axe de la roue par rapport à l'axe d'orientation. Ce système de réglage est simple et efficace; il a toutefois l'inconvénient de provoquer des à-coups, des chocs lors des brusques variations de vitesse de vent, la machine, sous l'action du ressort, revenant brutalement dans sa position de marche face au vent. On remédie très efficacement à cet inconvénient par l'emploi d'un amortisseur, du type amortisseur d'automobile, monté sur l'articulation de l'empennage; l'emploi d'un tel amortisseur est particulièrement recommandé pour les machines de grand diamètre.

Différents types de pompes peuvent être entraînés par l'éolienne lente. Etant donné le mode d'attaque par une tige descendant le long de la conduite de refoulement, et bien adaptée à des efforts de traction, c'est la pompe à simple effet qui est la plus avantageuse et de beaucoup la plus utilisée. Le fonctionnement de cette pompe, toutefois, a l'inconvénient de présenter une grande irrégularité du couple absorbé, puisqu'elle ne demande d'énergie que pendant un demi-tour de l'arbre manivelle, la descente du piston se faisant sous son propre poids.

La réduction de cette irrégularité serait intéressante, car elle a une répercussion directe sur le couple de démarrage, donc, en définitive, sur le temps de fonctionnement de la machine. On peut chercher à réduire l'irrégularité par l'emploi de pompe à double effet, mais la tige de commande du piston sera alternativement soumise à des efforts de traction de compression, un guidage de la tige s'impose pour éviter le flambage, et cette solution est rarement retenue.

Une autre combinaison avantageuse consisterait à utiliser une pompe à deux pistons dans un même corps, avec deux tiges de manœuvre; cette solution est très intéressante du point de vue de la régularité du couple absorbé, ainsi naturellement que de la régularité du débit, mais le mécanisme de commande est beaucoup plus compliqué, aussi n'est-il pas utilisé. Elle serait peut-être à prendre en considération dans le cas où la longueur des conduites de refoulement est importante.

La pompe à simple effet étant adoptée pour sa rusticité, on cherchera à régulariser le couple absorbé par un dispositif extérieur : levier, poids 

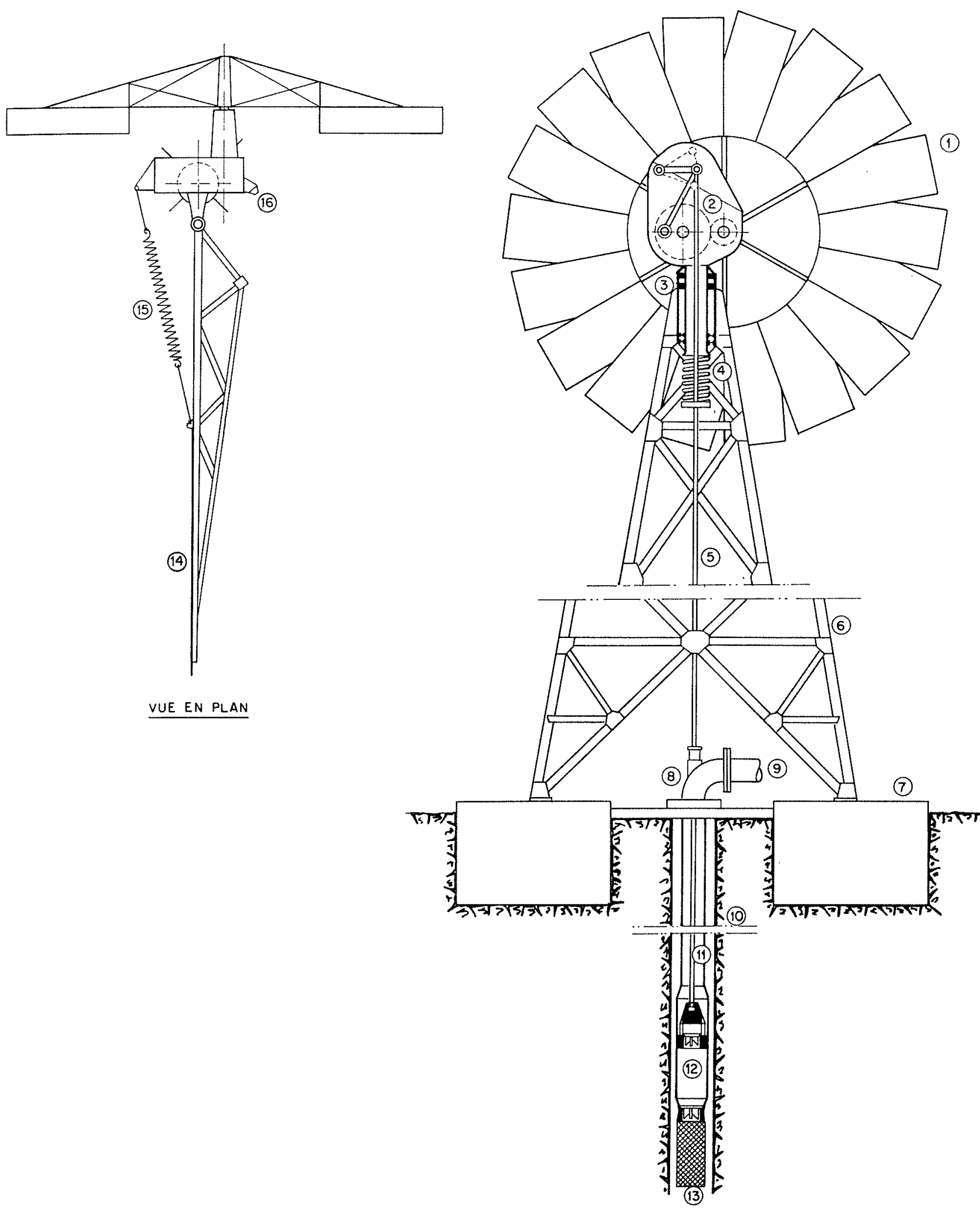

FIG. 15. - Mécanisme d'une éolienne lente pour la commande d'une pompe à piston.

Slow-running windmill mechanism driving a reciprocating pump.

ou ressort. Le système du ressort paraît être le plus avantageux car, en évitant des articulations supplémentaires, il permet de réduire les froltements parasites du mécanisme. L'emploi du 
système de compensation a deux effets favorables. Il permet ’̀ la machine de démarrer avec une vitesse de vent plus faible; il permet, en outre, de réduire les efforts dans le mécanisme de commande. L'irrégularité du couple absorbé par la pompe entraîne des variations cycliques de vitesse de la machine. Dans le cas d'une poimpe à simple effet sans compensation, pendant la montée de la tige, la roue fournit de l'énergie et ralentit; par contre, lors de la descente, le poids de l'équipage mobile fournit un couple moteur, la roue ne produisant plus d'énergie accélère; elle emmagasine de l'énergie cinétique qu'elle restitue à la course suivante. Les variations de vitesse de rotation qui en résultent sont malgré tout assez faibles, par suite de l'inertie de la roue; cette marche irrégulière est d'ailleurs sans effet nuisible.

Pour réduire l'effort au démarrage et éviter de démarrer avec la pleine charge sur le piston, on utilise parfois une fuite permanente au clapet de refoulement. Cette disposition présente des avantages et des inconvénients, comme on va le voir.

La fuite permanente établit une communication entre les deux faces du piston; schématiquement, on peut alors représenter la pompe
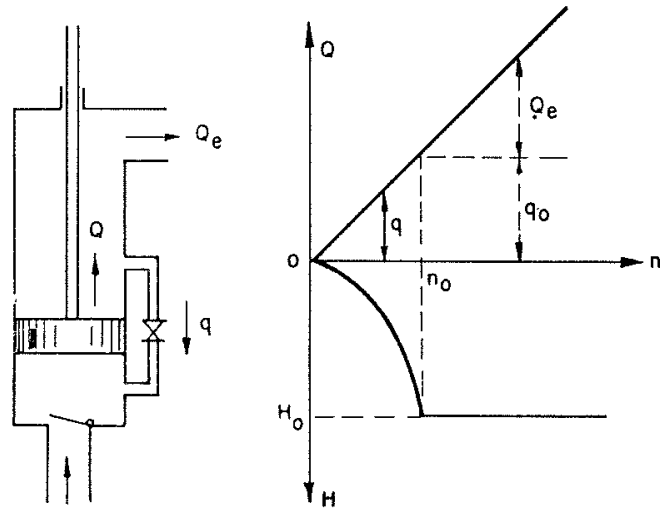

FIG. 16. -- Influence d'une fuite permanente sur le fonctionnement d'une pompe à piston.

Effect of a continuous leak on the operation of a reciprocating pump.

par le croquis ci-contre. Si on appelle $Q$ le débit de déplacement de la pompe (volume engendré par le déplacement du piston), $q$ le débit passant par la fuite et $Q_{e}$ le débit effectif passant par le tuyau de refoulement, on a :

$$
\mathrm{Q}=q+\mathrm{Q}_{e}=\frac{\pi d^{2}}{4} \mathrm{~S} \cdot \frac{n}{60}
$$

avec $d=$ diamètre du corps de pompe;

$\mathrm{S}=$ course;

$n=$ nombre de courses par minute.
Si $\omega$ est la section de fuite et $\mathrm{H}$ la pression engendrée par la pompe, on a :

$$
q=\omega \sqrt{2 g \mathrm{H}}
$$

Si $\mathrm{H}_{0}$ est la pression de refoulement de régime normal (valeur sensiblement constante, aux variations de niveau dans le réservoir près), le débit effectif sera nul tant que $H$ sera plus petit que $\mathrm{H}_{0}$, puis, lorsque la valeur $\mathrm{H}_{0}$ sera atteinte, le débit de fuite restera constant. On peut représenter sur un graphique en fonction du nombre $n$ de courses à la minute, les variations du débit et de la pression de refoulement. Le débit $Q$ varie linéairement. La pression $H$ varie suivant une parabole jusqu'à ce que la pression $H_{0}$ soit atteinte; elle reste alors constante. La pression $\mathrm{H}_{0}$ sera atteinte pour un nombre de courses $n_{0}$ à partir duquel le débit de fuite restera constant avec la valeur $q_{0}$; c'est à partir de $n_{0}$ seulement que la pompe débitera effectivement. La section de fuite dépend du nombre $n_{0}$ adopté suivant la relation :

$$
\omega=\frac{\pi d^{2}}{4} \mathrm{~S} \cdot \frac{n_{0}}{60} \frac{1}{\sqrt{2 g \mathrm{H}_{0}}}
$$

Mais du fait de la fuite permanente, le rendement de la pompe sera inférieur à l'unité. Indépendamment des autres pertes inévitables (clapets, pertes de charge, etc.) le rendement sera :

$$
\eta=\frac{\mathrm{Q}_{e}}{\mathrm{Q}}=\frac{\mathrm{Q}_{o}}{\mathrm{Q}_{e}+q_{0}}
$$

Il sera done nul pour $n=n_{0}$ et croitra ensuite suivant la relation indiquée. Cette perte de rendement est loin d'être négligeable, si on ne veut pas tomber sur une section de fuite si ridiculement petite qu'elle risque d'être bouchée par le moindre grain de sable; que la fuite soit bouchée, la chose n'est pas très grave, mais ce qui l'est davantage, c'est de voir la fuite agrandie par l'érosion (or cette fuite est toujours parcourue par un courant à grande vitesse), car le rendement tombe alors très rapidement.

Un exemple numérique fera mieux sentir la difficulté.

Prenons le cas d'une pompe répondant aux caractéristiques suivantes :

$$
\mathrm{H}_{0}=50 \mathrm{~m} \quad d=120 \mathrm{~mm} \quad \mathrm{~S}=385 \mathrm{~mm}
$$

Si on prend $n_{0}=3$, la fuite devra avoir une section de $0,072 \mathrm{~cm}^{2}$, soit un orifice circulaire d'un diamètre de $3 \mathrm{~mm}$ seulement.

Si la vitesse de régime normal est $n=15$, le rendement, du fait de la fuite, sera :

$$
\eta=\frac{15-3}{15}=80 \%
$$


Donc, en régime normal, $20 \%$ de la puissance de la machine seront absorbés par la fuite permanente. Certes, la machine démarre pour un vent plus faible que s'il n'y avait pas de fuite, mais, tout compte fait, le gain est assez illusoire. Peu importe en ellet que la machine démarre si elle ne pompe pas d'eau.

\section{Détermination des conditions de fonctionnement d'une éolienne lente avec pompe à piston}

L'éolienne est caractérisée par ses caractéristiques donnant le couple moteur ou la puissance en fonction de la vitesse de rotation pour un vent et un diamètre donnés; il est également intéressant, pour déterminer la résistance des organes et principalement du pylòne, de connaître la poussée axiale en fonction de la vilesse du vent; ces grandeurs sont généralement donnces sous forme de coefficient sans dimension.

Compte tenu des frottements du mécanisme de commande de la pompe et du rendement de la pompe à piston (frottement, fuites, pertes de charge), on obtient généralement un coefficient de puissance pratique ou rapport de l'énergie hydraulique obtenue à l'énergie cinétique tolale du vent, d'une valeur moyenne de 0,2 . Ce rendement assez faible se décompose de la façon suivante :

Rendement théorique d'une éolienne parfaite................

Rendement propre d'une roue lente. 0,55 à 0,60

Rendement de l'ensemble mécanisme, transmission, pompe, pertes de charge . . . . . ........

Il n'est pas inutile de rappeler que la notion de rendement énergétique est d'un intérêt secondaire; ce qu'il importe de connaitre par contre est le prix de revient de l'unité de volume de l'eau pompée.

Nous allons, en nous appuyant sur un exemple concret, déterminer la production d'une éolienne lente à piston, afin de déterminer le prix de revient du $\mathrm{m}^{3}$ d'eau pompée. Nous prendrons l'exemple d'une éolienne de $6,10 \mathrm{~m}$ de diamètre devant pomper de l'eau sous une hauteur de refoulement totale de $40 \mathrm{~m}$. La pompe à piston ćtant une pompe à couple constant (aux irrégnlarilés près, signalćes plus haut, mais en marche normale et compte lenu de l'inertic de la roue, lout se passe comme si la machine absorbait un couple constant), il est commode de reprísenter les caractéristiques de l'ćolienne sur un graphique couple-vitesse avec la vitesse du vent comme parametre. Il convient tout d'abord de choisir l'alésage el la course de la pompe. On considérera 4 modìles de pompes résultant de la combinaison de deux corps de pompe arec deux calages de manivelles, soit :

\begin{tabular}{|ll|ll|}
\hline alesage : & $125 \mathrm{~mm}$ & alésage : & $160 \mathrm{~mm}$ \\
course : & $384 \mathrm{~mm}$ & course : & $384 \mathrm{~mm}$ \\
cylindré : & 1,7 litres & cylindrèe : & 7,7 litres \\
\hline alesage : & $125 \mathrm{~mm}$ & alésage : & $160 \mathrm{~mm}$ \\
course : & $490 \mathrm{~mm}$ & course : & $490 \mathrm{~mm}$ \\
cylindrée : & 6 litres & cylindrée : & $9,9 \mathrm{litres}$ \\
\hline
\end{tabular}

L'intersection de l'horizonlale de couple constant de la pompe avec les courbes de couple à vitesse de vent constant de l'éolienne permettait d'établir la vitesse de rotation de la machine en fonction de la vitesse du vent pour les 4 modèles de pompe. On en déduit aisément les courbes donnant le débit en fonction de la vilesse du vent pour les 4 modìles de pompes. On remarquera que les 4 courbes obtenues ont sensiblement pour enveloppe une parabole cubique ror-

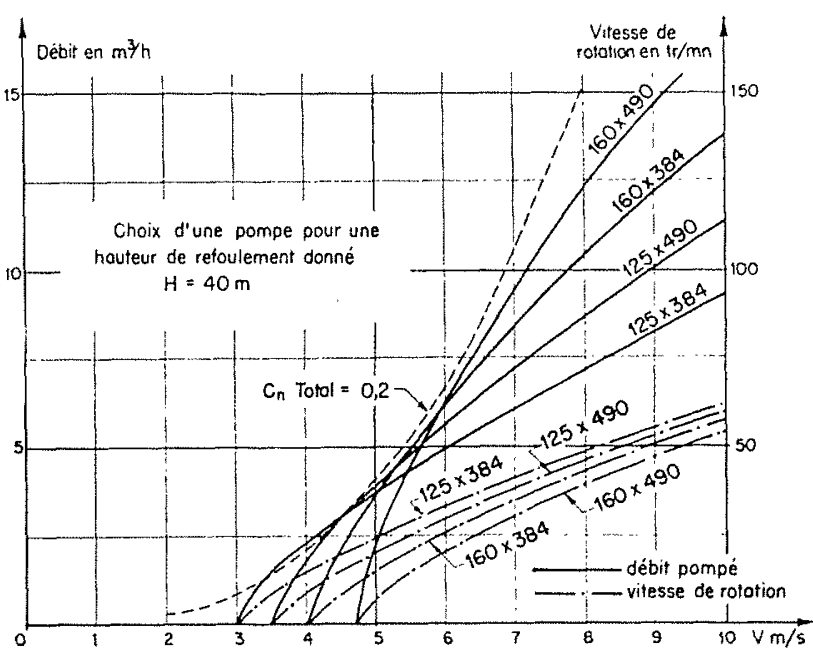

Fig. 17. - Choix d'une pompe à piston pour une éolienne lente de $6,10 \mathrm{~m}$ de diametre et pour une hauteur de refoulement de $40 \mathrm{~m}$. Courbes domnanl la vitesse de rolation et le débil pompé, an fonction de la vilesse du rent, sans fimitation par eclipsage.

Choice of a reciprocaling pump for a $6.1 \mathrm{~m}$ diamclet sloulu-running windmill and a discharge head of $10 \mathrm{~m}$ Curves give the speed of rolation and the discharge pumped, in lerms of wind speed, ignoring any limitation due to eclipsing.

respondant au coefficient de puissance 0,2 . Ies zones de tangence à l'enveloppe sont les zones de meilleur rendement.

On constate done que, suivant la vitesse du vent à équiper, il y aura lieu d'adopler tel ou 
lel type de pompe. La machine n’est pas appelée a fonctionner pour une seule valeur de la vitesse du vent, mais pour toute une gamme de vitesses, aree des durées caractérisées par la courbe vitesse-duréc de la région considérée. Partant de la courbe vilesse-durée et des courbes de débit en fonclion de la vitesse du vent, il sera facile de déterminer pour chaque type de pompe le volume lolal d'cau pompée dans l'année, ainsi que le nombre total d'heures de fonctionnement. On tiendra igalement compte de la vitesse du vent à partir de laquelle l'éclipsage de la roue commence à se produire; on admettra qu'à partir de cette vitesse, la puissance, donc le débit de la machine, est maintenue comstante. I.es résultats obtenus sont consignés dans un graphi-

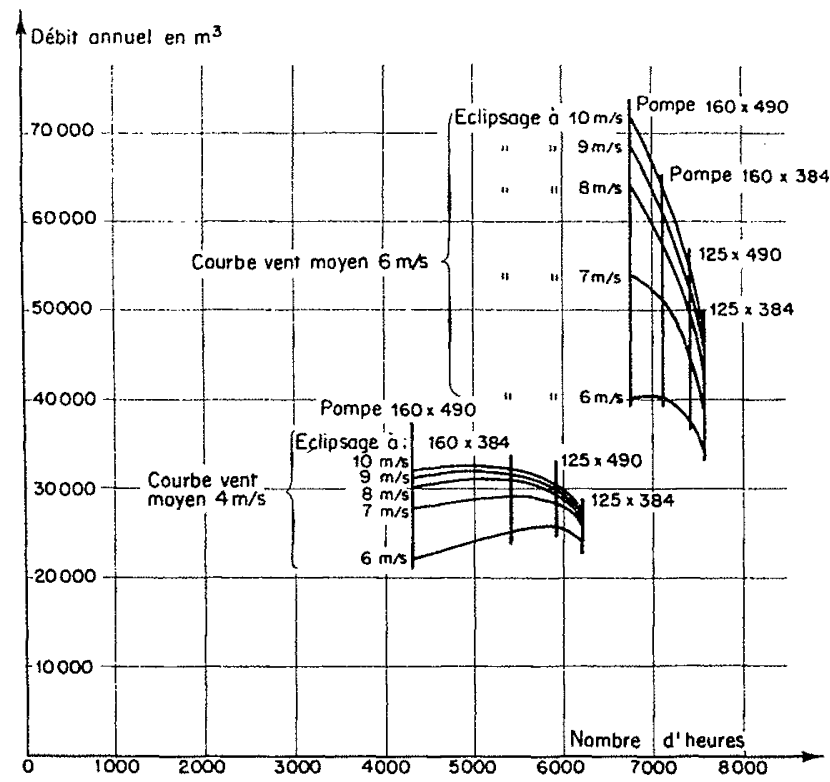

Fik. 18. - Gaphique d'utilisation d'une éolienne lente de $6,10 \mathrm{~m}$ aree pompe à piston refoulant à $40 \mathrm{~m}$.

Cilization graph for a $6.1 \mathrm{~m}$ sloun-running undmill with a reciprocating pump discharging under $40 \mathrm{~m}$.

que déhit annucl-nombre d'heures, ou graphique d'utilisation. Sur le graphique, on a porté les résultats obtenus en partant des courbes vitessedurée correspondant aux vitesses moyennes annuelles : $6 \mathrm{~m} / \mathrm{s}$ et $4 \mathrm{~m} / \mathrm{s}$. On conslate ainsi (que, par renl moyen de $4 \mathrm{~m} / \mathrm{s}$, il est possible de pomper jusqu'à $30.000 \mathrm{~m}^{3}$ par an sous une hauleur de $40 \mathrm{~m}$ avec une durée de fonctionnement de 6.000 heures, soit pendant $68 \%$ du temps. Par contre, arec un vent moyen annuel de $6 \mathrm{~m} / \mathrm{s}$, on alleindra $50.000 \mathrm{~m}^{3}$ par an avee une durée de fonctionnement de 7.500 heures, soit $85 \%$ du temps.

Enfin, moyennant une réduction de la duréc de fonctionnement, il sera possible d'aceroitre le débit pompé jusqu'à $72.000 \mathrm{~m}^{3}$ par an. Elant donné l'inlérêt qu'il y a, pour ee lype d'installattion, à obtenir le maximum de temps de fonctionnement, considérons seulement le point correspondant à $50.000 \mathrm{~m}^{3}$ et 7.500 heures. Le pompage de $50.000 \mathrm{~m}$ " par an à $40 \mathrm{~m}$ représente une énergie hydraulique équivalente à $5.500 \mathrm{kWh}$ par an. Si l'colienne étail remplacée par un groupe de pompage électrique, pour lequel il faudrait admettre un rendement total de 0,53 , soit :

Rendement du moteur . . . . . . . . . 0,80

Perte en ligne ............. 0,05

Rendement total de la pompe, transmis-

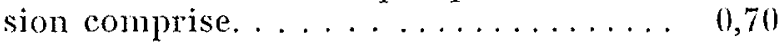

on serait conduit à une consommation au compteur de $10.400 \mathrm{kWh}$ par an. Dans le cas d'un pompage par moteur à combustion interne, on obtiendrait, dans les mèmes condilions, une consommation annuelle de 4.1001 de carburant.

Sans tenir compte des frais d'inslallation de la ligne dans le cas de l'épuipement ílectrique, ni des frais de surveillance et d'entretien dans le cas du groupe thermique, une lelle installation eolienne serait amorlie en moins de 7 ans, alors qu'on peut compter sur une durée de vie normale de 20 ans en cas d'entretien convenable. Les frais occasionnés par la ligne et par la présence d'un surveillant pour le moleur thermique devenant considérables lorsque le groupe de pompage est éloigné d'un centre luabité, la rentabilité de l'éolienne est notablement accrue dans ces conditions, en sorte que celte solution peut devenir de beaucoup la plus avantageuse.

\section{Eoliennes rapides pour pompes centrifuges ou à hélices}

On appelle «éolienne rapide» une machine à faible nombre de pales dont la vitesse de rolalion correspond à un rapport $u / v$ périphériquc de l'ordre de 3 à 7 ou 8 el mêne plus. Ces machines présentent des caractéristiques de fonctionnement très différentes de celles des éoliennes lentes. La variation du coeflicienl do couple en fonction du rapport $u / v$ est la suirante : au démarrage, le couple est tris faible, puis croît en même temps que le rapport $u / n$ jusquà alteindre un maximum qui se situe sensiblement dans la zone de rendement maximum. A partir de ce maximum, le couple décroîl continuellement lorsque le lapport $u / v$ augmente jusqu'à l'emballement. La zone de couple croissant entre le démarrage el le maximum correspond souvent à des instabilités de fonctionnement. Le trìs faible couple de démarrage de ees machines les rend inaples à l'entraine- 
ment direct de pompes à piston. Un tel genre de pompe ne peut être entrainé convenablement qu'en inlerposant, entre l'éolienne et la pompe, soit une transmission électrique, soit un accouplement à force centrifuge permettant à l'éolienne de prendre de la vitesse pour atteindre un régime à couple moteur suffisant.

Par contre, les pompes centrifuges ou hélices s'adaptent remarquablement bien à l'accouplement à de telles éoliennes, car leur couple de démarrage est très faible et leur caracléristique théoriquement possible d'oblenir une bonne ulilisation de l'éolienne lente et une bonne production d'énergie avee les groupes a pompe à piston, mais ceci entraînerait des complications mécaniques, comme une variation automatique du rapport de réduction éolienne-pompe, complicalions qui ne s'accordent pas avec la ruslicilé exigée de ces machines de petite puissance. II semble donc bien, et ceci pour des raisons d'économie el d'exploitation, que l'ulilisalion de l'éolienne lente restera limilée aux petites puis-

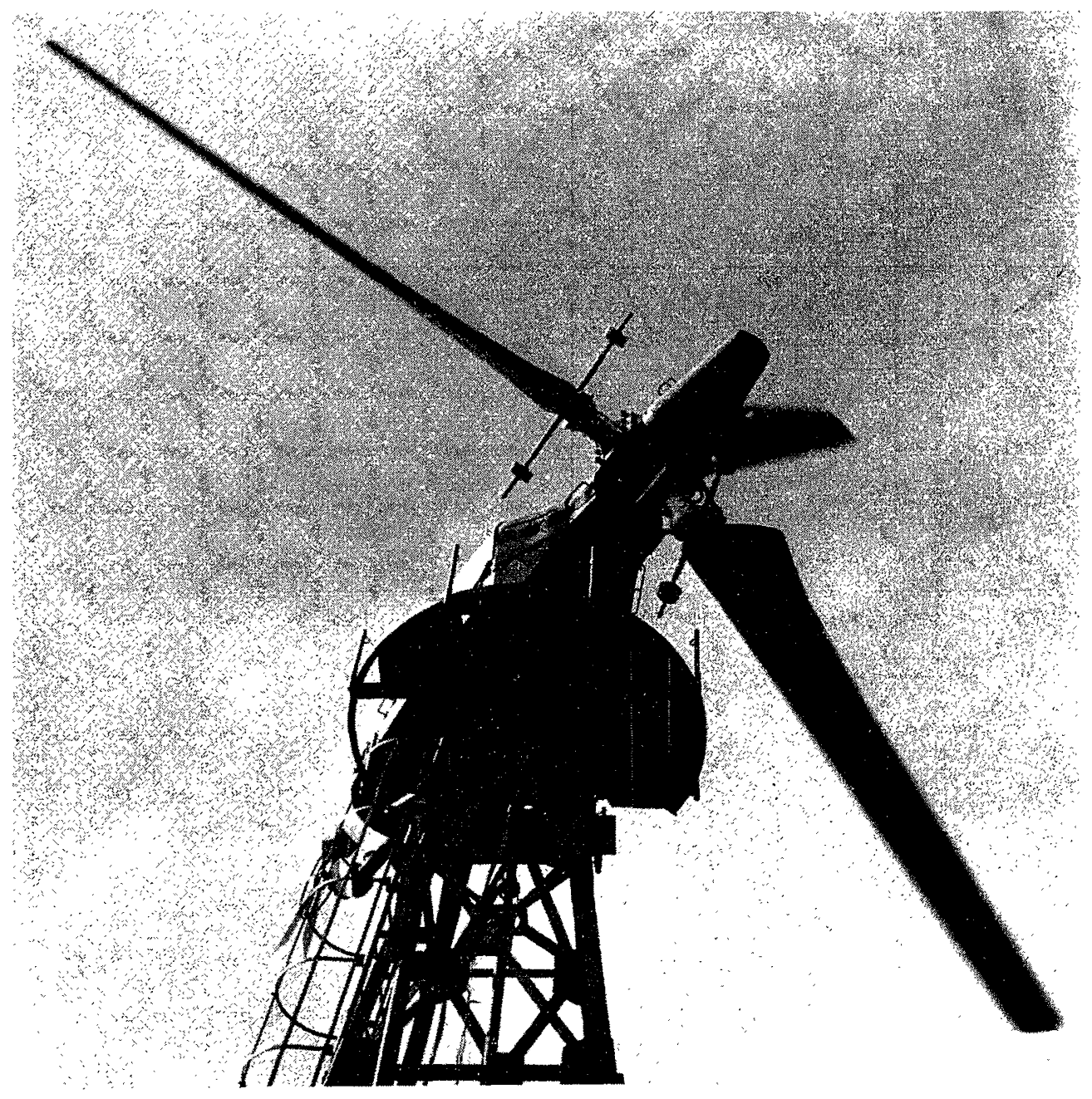

Fra. 19. - Eolienne rapide pour pompe centrifuge avec rouc de 8 m de dinmetre à pales orientables à liaison elastique. Construction Neyrpic.

$8 \mathrm{~m}$ diumeter, high speed windmill, wilh spring linked adjustuble bludes, for a centrifugal pump. Manufarlured by Neyrpic.

puissance-rilesse est telle qu'il est possible de faire coïncider les zones de fonctionnement à rendement maximum de l'éolienne et de la pompe. On ne sera done pas élonné d'obtenir avec ces groupes une production hydraulique toujours nettement plus ćlevée qu'avec les groupes à pompe à piston pour un même diamètre de roue et un même site. Certes, il esl sances, cependant que l'éolienne rapide verra son champ d'application s'étendre dans le domaine des moyennes et grandes puissances, pour lesquelles un bon rendement global devient nécessaire.

Les coliennes rapides peuvent clre de deux types : soit à pales fixes, soit à pales orientables. La machine à pales fixes utilise pour son réglage 


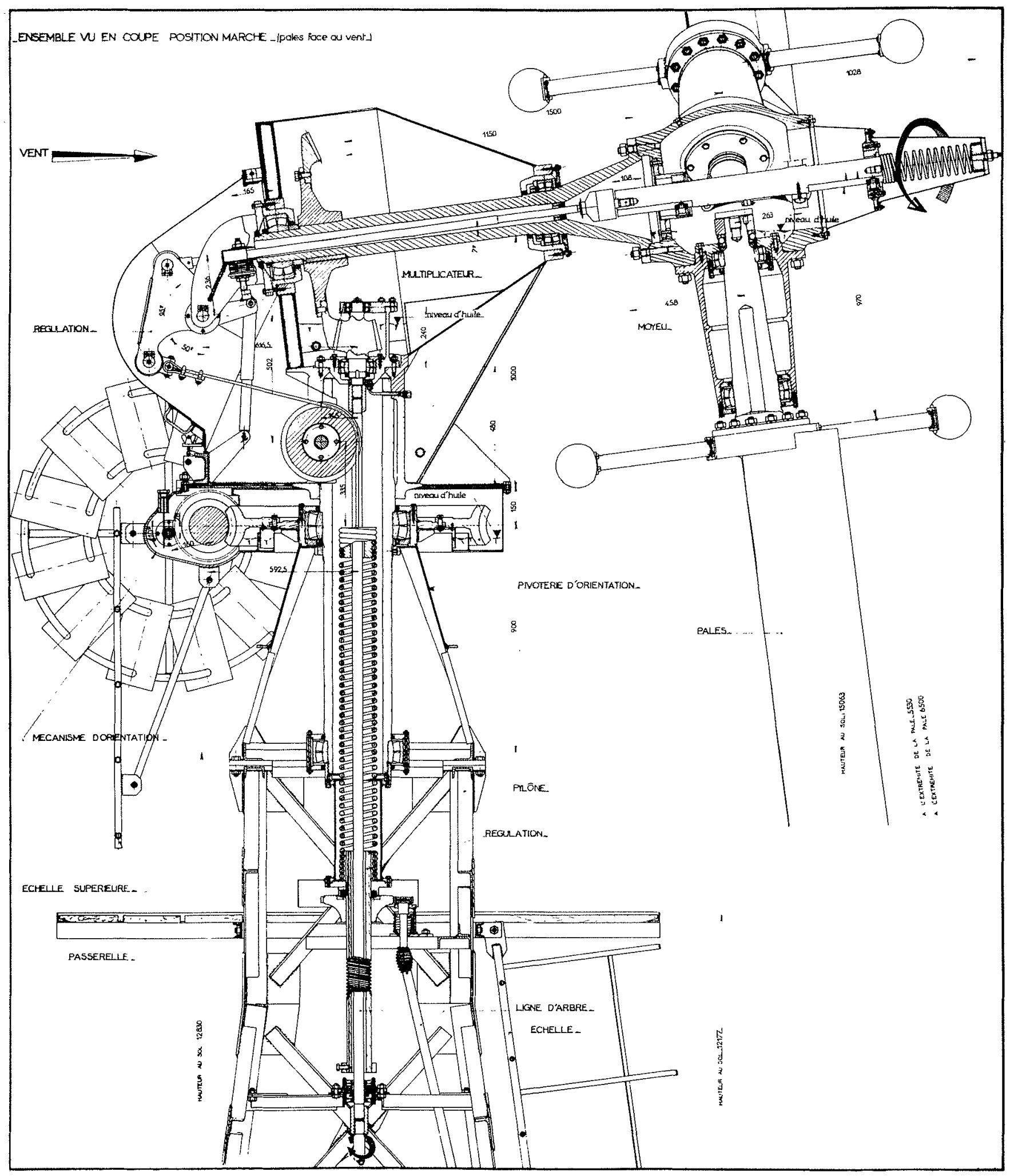

Far. 20. - Mécanisme d'une éolienne rapide de $13 \mathrm{~m}$ de diamètré a pales orientables à liaisons álastiques.

Mechanism of a 13 m diumeler high speed windmill with spring hinked adjuslable blades. 


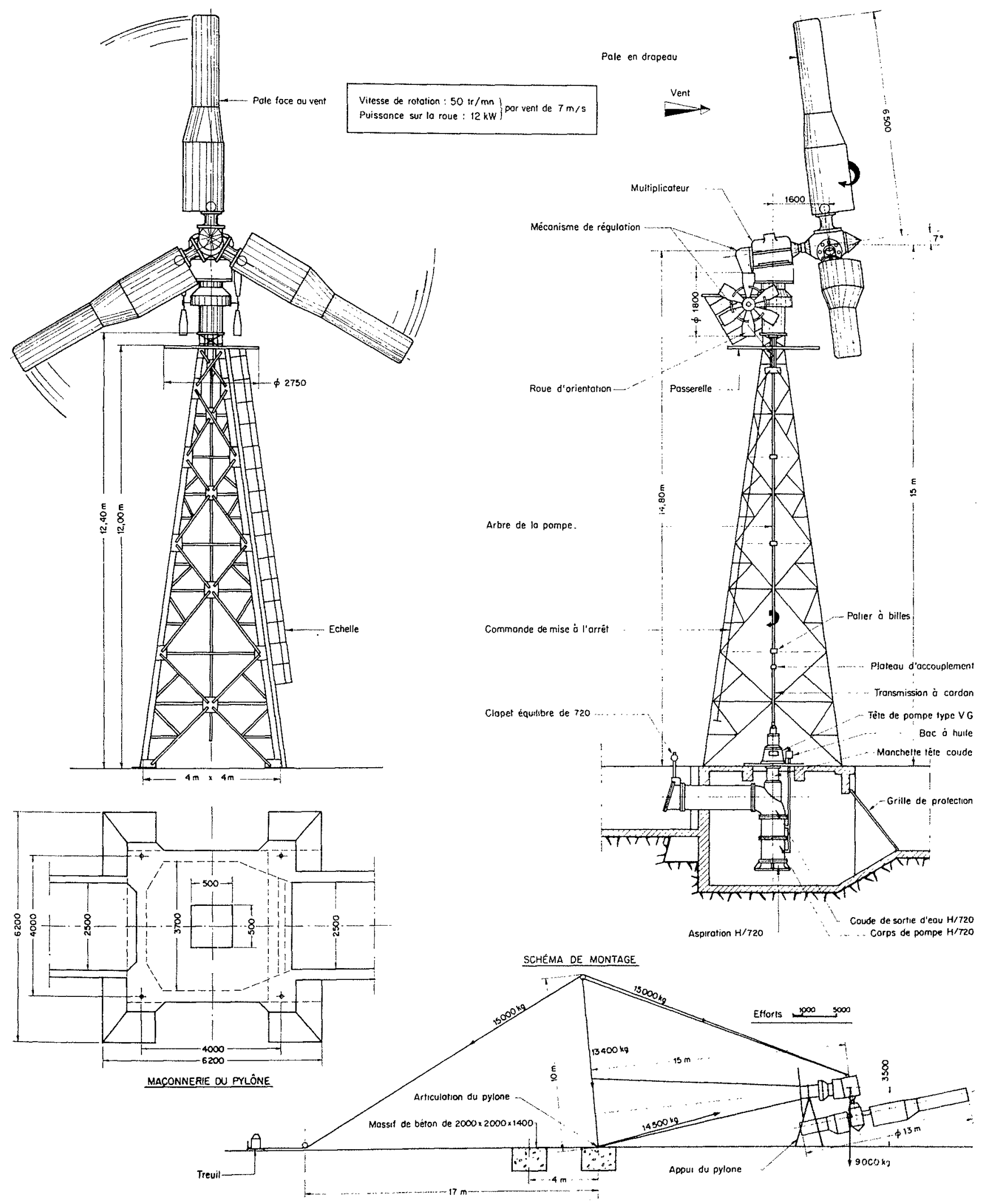

Fra. 21. - Eolienne mide Neyrpic de $13 \mathrm{~m}$ de diamètre entrainant une pompe it hẻlice Alta pour l'irrigation.

Neyrpic $13 \mathrm{~m}$ diameler, high speed windmill driving an Alta propeller pump for irrigation purposes. 
une propriété remarquable des profils fins. Lorsque l'angle d'incidence du courant relatif par rapport à la pale atteinl une certaine valeur, le coefficient de portance du profil décroit brusquement, et la puissance de la machine est limitée au lieu de croître comme le cube de la vitesse du vent. Ce phénomène, bien connu en aviation sous le nom de perte de vitesse, peut être utilisé pour limiter la puissance de l'éolienne; il nécessite toutefois l'emploi de pales très fines, dont la tenue aux tempêtes et aux brusques rafales est difficile à assurer.

La machine à pales orientables permel une plus grande souplesse de fonctionnement et résout heureusement le problème de la tenuc par grand vent. Le fonctionnement de celle machine est le suivant : - pour les vitesses de vent comprises entre la vitesse de démarrage el la vitesse nominale, le calage des pales reste constant; la machine se comporte comme une machine à pales fixes dont le régime est défini par l'intersection des caractéristiques ćolienne et pompe. Lorsque la vitesse nominale est dépassée, le mécanisme de réglage intervient pour modifier l'inclinaison des pales et maintenir constantes la puissance et la vitesse. Différents systèmes peuvent être utilisés pour commander l'orientation des pales : force centrifuge, poussée aérodynamique sur les pales, etc..., ou même, pour les machines de grande puissance, servo-moteurs et régleurs. Le réglage utilisant un système de ressort équilibrant la poussée aérodynamique des pales présente le gros avantage de se comporter remarquablement bien aux rafales de vent, car sous l'effet d'une brusque augmentation de vitesse du vent, les pales cèdent en limitant à la fois la vitesse de rotation et l'effort subi par l'ensemble de la machine. La figure 21 représente une telle machine accouplée à une pompe hélice; dans cette machine, l'axe de tourillonnement des pales est choisi de telle manière que la résultante aérodynamique des efforts sur la pale crée un couple toujours de même signe; ce couple, croissant avec la vitesse du vent, est équilibré par un système élastique choisi de telle facon que la vitesse de rotation soit approximativement constante dans la zone de réglage et tende à diminuer pour les très grands vents, les pales se mettant alors en drapeau. Le moment d'inertie de la pale autour de son axe de tourillonnement étanl très faible, le système élastique cède immédiatement sous l'effet d'une rafale et les efforts supportés par l'ensemble de la construction sont réduits. La valeur de la vitesse du vent à partir de laquelle le réglage intervient, ainsi que celle à partir de laquelle la machine se met en drapeau, peuvent s'ajuster par le réglage de la tension initiale du ressort. L'arbre de la roue entraine un couple conique mulliplicateur alta- quant un arbre vertical situé sur l'axe du pylòne et relié au sol à la pompe, elle-même à axe vertical, ainsi qu'il convient pour une pompe noyćc. L'orientation de l'ensemble du mécanisme autour d'un axe vertical est assurée par une roue auxiliaire; celle-ci, par l'intermédiaire d'engrenages démultiplicateurs, altaque une vis globique irréversible. Ce dispositif très efficace joue à la fois le ròle de servo-moteur et de limiteur de vitesse d'orientation, pour éviter les efforts gyroscopiques trop considérables.

\section{Détermination des conditions de fonctionnement d'une éolienne rapide avec pompe centrifuge}

Deux régimes de marche doivent être considérés pour l'éolienne: lors de la marche pour vitesse de vent supérieure à la vitesse nominale, le système de réglage intervenant, la puissance fournie par la machine est maintenue sensiblement constante, et le groupe fonctionne dans les mêmes conditions qu'un groupe pompe normal à moteur électrique. Par contre, pour les vitesses de vent inférieures à la vitesse nominale, le système de réglage n'intervient pas et la machine fonctionne comme une éolienne à pales fixes.

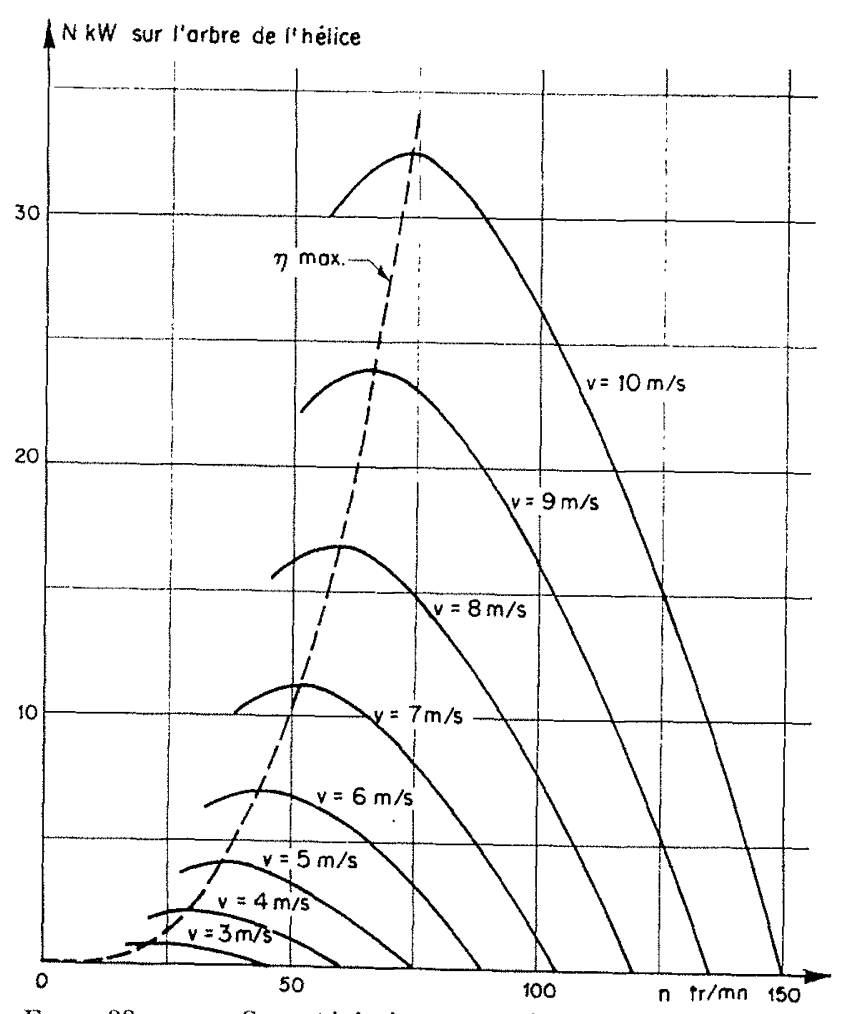

Fic. 22. - Caractéristiques puissance-vitesse d'une colienne rapide de $13 \mathrm{~m}$, pour différentes valeurs de la vilesse du vent $\left(\beta=10^{\circ}\right)$.

Power-speed characteristics of a $13 \mathrm{~m}$ high speed mindmill for various values of wind speed $\left(\beta=10^{\circ}\right)$. 
C'est ce mode de fonctionnement que nous examinerons particulièrement.

Alors que dans le cas des éoliennes pour pompe à piston, il était commode de représenter les caractéristiques de l'éolienne dans un diagramme couple-vitesse de rotation, car la pompe à piston était une machine à couple constant, ici, par contre, étant donné la facon dont sont données habituellement les caractéristiques des pompes, il sera préférable de représenter les conditions de fonctionnement de l'olieme dans un diagramme puissance-vitesse de rotalion. Dans un tel diagramme, et pour une vitesse de vent donnée, la variation de la puissance disponible sur l'arbre de l'ćolienne, en fonclion de la vitesse de rotation, est représentée par une courbe d'allure parabolique. La partic gauche de cette courbe n'est généralement pas utilisée ef correspond à des instabilités de régime surtout pour les machines rapides. Le sommet de la courbe correspond à la zone de rendement maximum; l'emballement s'obtient pour une vilesse de rotation sensiblement double de celle du rendement maximum. On peut passer arec une bonne approximation d'une courbe correspondant à une valeur $v_{1}$ de la vitesse du rent à une courbe correspondant à la valeur $\nu_{2}$ en multipliant les abscisses par le rapport $\bar{v}_{2} / v_{1}$ et les ordonnées par le rapport $\left(v_{2} / v_{1}\right)^{3}$. Les points de rendement maximum sont done sensiblement situés sur une parabole cubique $\mathrm{N}=k n^{3}$.

Cette propriété, commune à toutes les turbomachines, appartient également aux pompes centrifuges et hélices; aussi faut-il s'attendre à une heureuse concordance des zones de fonctionnement à bon rendement de l'éolienne et de la pompe.

Les caractéristiques des pompes sont hahituellement données sous la forme de courbe débithauteur de refoulement, à vitesse de rolation constante. Pour l'étude de l'accouplement à unc colienne, il est plus commode d'utiliser les caractéristiques puissance absorbée-ritesse de rotation à hauteur de refoulement constante, cette dernière étant, en effet, pratiquement constante dans la plupart des cas. Si toulefois les pertes de charge du circuit sur lequel débite la pompe n'étaient pas négligeables, il conviendrait d'utiliser la courbe puissance-vitesse de rotation correspondant à la caractéristique débithauteur du circuit d'utilisation. Le passage des caractéristiques débit-hauteur à vitesse constante aux caractéristiques puissance-vitesse de rotation à hauteur constante se fait sans difficulté par les formules classiques de similitude, soit pour une même roue, soil pour des roues géométriquement semblables. II sera commode enfin de graduer les courbes puissance-vilesse a hauteur constante en débits. En superposant dans le plan puissance-vitesse de rotation les caractéristiques pompe et éolienne, on obtiendra les intersections définissant les points de fonctionnement et, pour chaque vitesse de vent, sera ainsi définie la vilesse de rotation et le débit fourni par la pompe sous la hauleur considérée.

Un type de pompe étant donné, la détermination de la courbe puissance-vitesse à hauteur constante nécessite tout d'abord la comnaissance du point de fonctionnement nominal. Sur l'éolienne, ce point sera défini par la vitesse de vent et la vitesse de rolation. On choisit généralement le point de rendement maximum sur la courbe $v_{n}=$ Cte. Ce point étant choisi, on connaît la puissance disponible et la vitesse de rotation. Reste à déterminer la pompe. Le type de machine étant connu et caractérisé, par exemple par une courbe $\mathrm{Q}-\mathrm{H}$ à $n=$ Cle pour une machine de diamctre $\mathrm{D}_{1}$, on choisira sur la courbe un point de fonctionnement $\left(Q_{1}, H_{1}\right.$, $n_{1}, \mathrm{~N}_{1}$ ). On cherchera alors, par les formules habituelles de similitude, te diametre et la vitesse de rotation qu'il convient de donner à celle pompe pour qu'elle absorbe la puissance $\mathrm{N}_{2}$ sous la hauteur $\mathrm{H}_{2}$.

$$
\text { Soit : } A=\mathrm{H}_{2} / \mathrm{H}_{1} \quad \text { et } \quad B=\mathrm{Q}_{2} / \mathrm{Q}_{1}
$$

On a $N_{2} / N_{1}=A$. B. D'où le rapport $B$ définissant le débit de la pompe. Le diametre et la vitesse sont alor's donnés par :

$$
\frac{D_{2}}{D_{1}} \cdots \frac{B^{1 / 2}}{A^{1 / 4}}
$$

et :

$$
\frac{N_{1}}{N_{1}}: \frac{A ; 1}{B^{1 / 2}}
$$

La connaissance de $n .$, permet entre autres de définir le rapport de multiplication à prévoir entre l'hélice et la pompe. La pompe est alors déterminée par les grandeurs $D_{2,}, n_{2}, Q_{2}$. La courbe $\mathrm{N}-n$ à $\mathrm{H}_{2}=$ Cle recherchée n'est autre que la transformée à l'horizontale $\mathrm{H}_{1}=$ Cte de la colline de la pompe de diametre $\mathrm{D}_{1}$.

Le choix du point définissant le fonclionnement de la pompe est évidemment arbitraire. Pour les groupes à entrainement par moteur à vitesse constante, on choisit un point dans la région de meilleur rendement de la pompe. Dans le cas d'une pompe entraînée par une éolienne, il n'est nullement évident a priori que te point de rendement maximum de la pompe soit je plus favorable. Il faut en effet considérer ici que, d'une part, la production tolale d'énergie dépend de la courbe vitesse-durce du site considerri el, d'autre part, que te nombre total d'heures de fonctionnement est d'une srande importance 
pour le calcul des dimensions du réservoir d'accumulation.

\section{Influence du choix des points de fonctionnement de l'éolienne et de la pompe sur la production}

Pour mettre en lumière l'influence considé. rable du choix du point de fonctionnement de la pompe sur la production du groupe, nous nous appuierons sur un exemple concret. On étudiera l'accouplement d'une ćolienne rapide de $13 \mathrm{~m}$ de diamètre, dont les caractéristiques puissancevilesse sont données par la figure 22 avec une pompe centrifuge dont les caractéristiques débithauteur sont données par la figure 23, la hauteur de refoulement étant de $40 \mathrm{~m}$ et les conditions

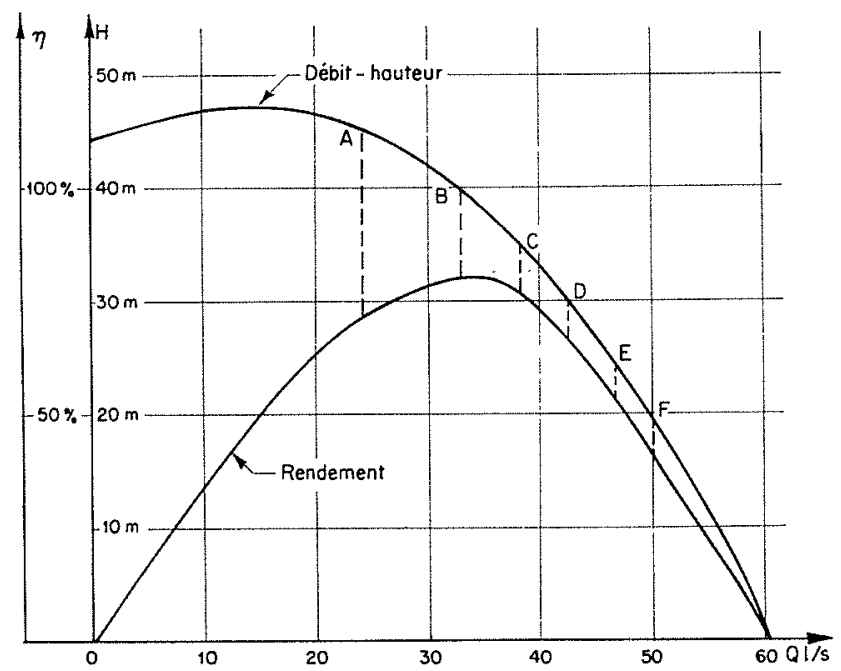

FIg. 23. - Caractéristique à vitesse constante d'une pompe centrifuge pour éolienne. Les différents points de fonctionnement $\mathrm{A}, \mathrm{B}, \mathrm{C}, \mathrm{D}, \mathrm{E}, \mathrm{F}$ se retrouvent sur les figures 24 et 25 .

Constant speed characteristics of a centrifugal pump for windmill application. The various operating points $A$ $B, C, D, E, F$ are repeated on Figs. 24 and 25.

météorologiques étant définies par la courbe vitesse-durée de la figure 7 correspondant à la vitesse moyenne $6 \mathrm{~m} / \mathrm{s}$. Le régime de marche est défini par le point nominal de l'éolienne et le point de fonctionnement correspondant de la pompe.

- Pour l'éolienne, on examinera successivement les points suivants : marche à rendement maximum pour les vitesses de vent : $6,7,8$ et $9 \mathrm{~m} / \mathrm{s}$; au-delà de ces vitesses, le réglage maintient la puisance constante.

- Pour la pompe, on examinera les points marqués A, B, C, D, E, F sur le diagramme et correspondant respectivement aux valeurs suivantes du rendement: $72,80,76,66,54$, $45 \%$. Au total, 24 régimes différents seront examinés. Pour chacun d'eux, on établira ainsi qu'il a été dit plus haut, une courbe puissance-vitesse de rotation pour $\mathrm{H}=40 \mathrm{~m}$ et la courbe correspondante donnant le débit en fonction de la vitesse du vent.

La figure 24 représente les courbes débilvitesse du vent pour les différents points de fonctionnement de la pompe, et pour la vitesse nominale $v==8 \mathrm{~m} / \mathrm{s}$. On constatera entre autres que l'amorçage de la pompe se fait pour un débit non nul; ceci est dû au fait que la courbe débithauteur à vitesse constante présente un maximum. Si cette courbe ne présentait pas de maximum, l'amorcage se ferait à débit nul, comme on le verra pour les autres types de pompes examinés plus loin.

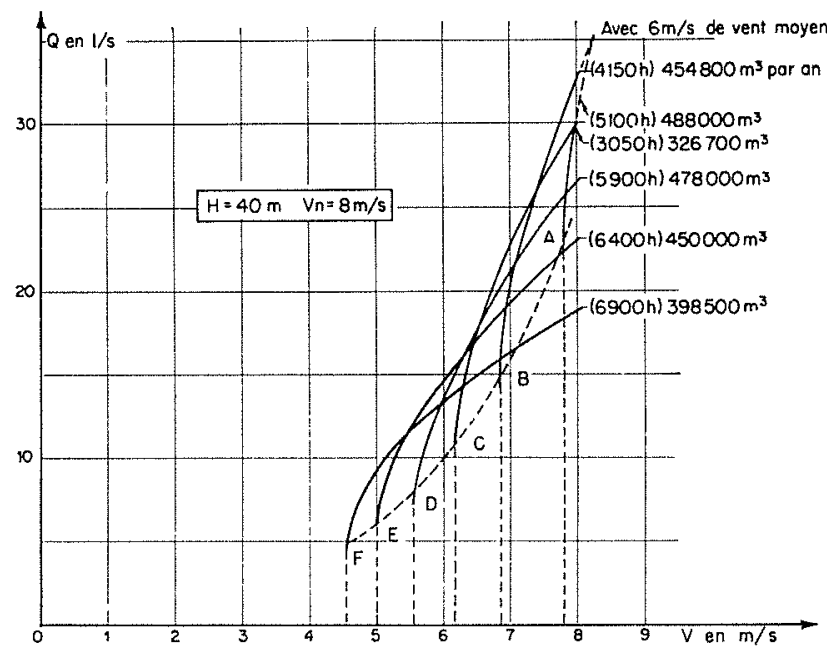

FIG. 24. - Courbes de débit en fonction de la vitesse du vent pour une pompe centrifuge refoulant sous $40 \mathrm{~m}$ et entraînée pour une éolienne de $13 \mathrm{~m}$ ayant une vitesse nominale de $8 \mathrm{~m} / \mathrm{s}$ et pour différents points de fonctionnement de la pompe.

Discharge curves against wind speed for a centrifugal pump worling under a head of $40 \mathrm{~m}$ and driven by a $13 \mathrm{~m}$ windmill having a rated wind speed of $8 \mathrm{~m} / \mathrm{sec}$. Curves taken for different operating points of the pump.

La courbe vilesse-durée définissant le régime météorologique fournit, pour chaque vitesse de rent, le nombre d'heures de fonctionnement, et permet, en s'appuyant sur la courbe débit-vitesse de vent, de calculer, pour une année, le volume total d'eau pompée et le nombre d'heures total de fonctionnement.

Chacun des 24 régimes de fonctionnement envisagé conduit ainsi à un ensemble de chiffres résumant la production annuelle. Ces résultats sont consignés dans un diagramme débit totalnombre d'heures; les différents points sont groupés suivant des courbes d'égales vitesses de vent nominales et d'égales conditions de fonc- 
tionnement de la pompe. L'examen du graphique ainsi obtenu fait parfaitement ressortir l'influence considérable du choix des points de fonctionnement sur la production. On constate, en particulier, que les régimes correspondant au rendement maximum de la pompe au point nominal sont loin de donner les meilleurs résultats; il convient, au contraire, d'adopter pour la pompe un point de fonctionnement au régime nominal situé très à droite du rendement maximum. C'est ainsi que, dans le cas considéré, le volume pompé maximum est obtenu pour tin rendement nominal de la pompe de $66 \%$, et la durée de fonctionnement maximum pour un rendement de pompe au point nominal de $45 \%$ seulement. L'établissement d'un projet sérieux de pompage par éolienne doit comporter l'élablissement de telles courbes, qui seules permet-

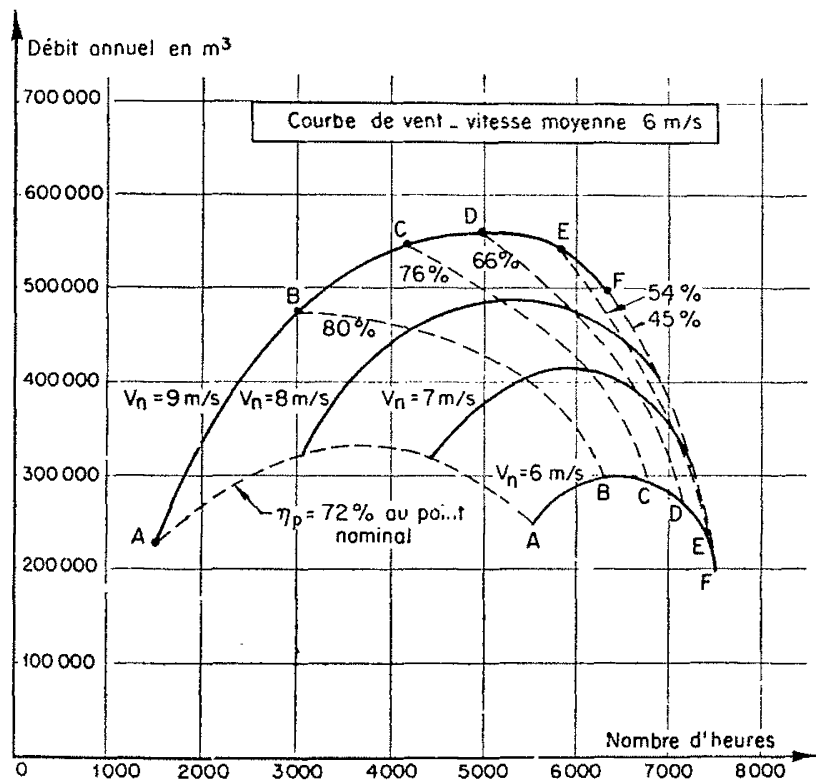

Fra. 25. - Graphique d'utilisation d'une éolienne rapide de $13 \mathrm{~m}$ avee pompe centrifuge refoulant sous $40 \mathrm{~m}$, pour une vitesse de vent moyenne annuelle de $6 \mathrm{~m} / \mathrm{s}$.

Utilization graph of a $13 \mathrm{~m}$ high speed windmill with a centrifugal pump discharging mander $40 \mathrm{~m}$ hend, for a annual mean wind speed of $6 \mathrm{~m} / \mathrm{sec}$.

tent de choisir correctement les caractéristiques de l'installation pour tenir compte tant des besoins en volume total qu'en nombre d'heures de marche.

Partant des chiffres qui viemnent d'être obtenus, on peut se faire une idée de la rentabilité de l'installation; considérons le point de fonctionnement caractérisé par une production annuelle de $550.000 \mathrm{~m}^{3}$ a $40 \mathrm{~m}$ et 5.750 heures de marche. Si le pompage était effectué au moyen d'un groupe pompe à moteur électrique, en admettant les rendements moyens suivants:
.... perte en ligne......... $5 \%$

... rendement du moteur . . . . $90 \%$

- rendement, transmission et pompe ............ $70 \%$

on serait conduit à une consommation annuelle de $107.000 \mathrm{k} \mathrm{W}^{\prime} \mathrm{h}$. Si le pompage était effectué au moyen d'un groupe thermique, la consommation annuelle de carburant serait de 34.000 litres. Dans ces conditions, et sans tenir compte des frais d'installation de la ligne d'une part, et des frais d'entretien et de surveillance, d'autre part (Crais qui peuvent être élevés si la pompe est éloignée d'un centre habité), l'amortissement de l'éolienne est assuré en quatre ans. L'installation d'un groupe de pompage arec éolienne est donc particulièrement intéressante. Ces conditions, déjà très favorables, peuvent être encore améliorées par l'emploi d'autres types de pompes, comme nous allons le voir.

\section{Influence du type de pompe sur la production}

Un facteur essentiel de la qualité d'un groupe de pompage par ćolienne est l'importance du nombre d'heures de fonctionnement. Pour accroître ce nombre d'heures, on vient de voir qu'il y avait intérêt à choisir un point de fonclionnement nominal de la pompe situé très à droite des points habiluellement choisis pour les groupes à moteur électrique ou thernique. Ceci est dû au fail cụe la caracléristique débithauteur d'une pompe centrifuge est très plate dans sa partie gauche entre le débil nul et le rendement maximum. Il serait avantageux de pouvoir utiliser des pompes dont la caractéristique débit-hauteur soit montante, même aux faibles valeurs du débit. C'est le cas des pompes à grande vitesse spécificue el particulièrement des pompes hélices. Alors que, sur une pompe centrifuge, la hauteur de refoulement à débit nul est de l'ordre de 1,2 fois la hauteur à rendement maximum sur une courbe à vitesse constante, par contre, pour la pompe à hélice, il n'est pas rare d'obtenir une hauteur de refoulement à débit nul double de celle du rendement maximum.

La pompe à hélice parait done mieux adaptée que la pompe centrifuge à l'entrainement par colienne. Toutefois, te gain sur le nombre d'heures de fonctionnement est moins grand qu'on ne pourrait s'y altendre, car la puissance absorbée à vitesse constante croît lorsque te débit diminue. On peut remédier à cette difficulté par l'emploi d'une pompe à pales orientables dont le réglage serait tel que l'inclinaison des pales diminuerait pour les faibles vilesses 
de venl; comme la puissance absorbée, toutes choses égales d'ailleurs, décroît lorsque l'inclinaison des pales diminue, on peut corriger l'efret reproché à la pompe-hélice et augmenter d'autant to nombre d'heures de fonctionnement.

Un exemple concret fera ressortir les particularités des trois types de pompe. On considere une éolienne de $13 \mathrm{~m}$ possédant les caracterisliques de la figure 22 et entraînant une ponpe devant refouler sous une hauteur de $4 \mathrm{~m}$. Le point nominal sera choisi pour $v=8 \mathrm{~m} / \mathrm{s}$ et la courbe vitesse de vent-durée correspond à unc vitesse moyenne annuelle de $6 \mathrm{~m} / \mathrm{s}$. Les pompes examinées seront les suivantes :

\section{1" Pompe centrifuge:}

Déduite par similitude de la pompe exaninée précédemment el utilisée au point nominal avec $n=80 \%$.

\section{2" POMPE HÉLICE:}

Angle de calage $\mathrm{B}=21^{\circ}$ et caraclérisée par les chifrres suivants pour marche à vitesse constante :

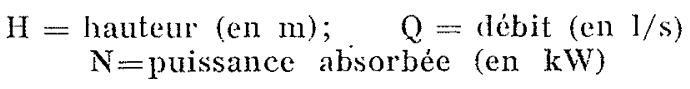

\begin{tabular}{|c|c|c|c|c|c|c|c|c|c|c|c|}
\hline $\mathrm{H}$ & 2 & 3 & 4 & 5 & 6 & 7 & 8 & 10 & 12 & 14 \\
\hline $\mathrm{Q}$ & 390 & 370 & 340 & 315 & 275 & 150 & 120 & 80 & 10 & 0 \\
\hline $\mathrm{N}$ & 12,5 & 14 & 17 & 20 & 22,5 & 25 & 27 & 32 & 37 & 42 \\
\hline
\end{tabular}

Soit, pour marche à hauteur de refoulement conslante :

$H=$ hauleur $(\mathrm{en} \mathrm{m}) ; \quad \mathrm{Q}=$ débil $(\mathrm{en} 1 / \mathrm{s})$ $\mathrm{N}=$ puissance absorbeje (en kW)

\begin{tabular}{|c|c|c|c|c|c|c|c|c|c|c|c|}
\hline $\mathrm{H}$ & 4 & 4 & 4 & 4 & 4 & 4 & 4 & 4 & 4 & 4 \\
\hline $\mathrm{Q}$ & 550 & 425 & 340 & 280 & 225 & 112 & 85 & 50 & 23 & 0 \\
\hline $\mathrm{N}$ & 35 & 21,5 & 17 & 14 & 12,2 & 10,5 & 9,6 & 8,2 & 7 & 6,3 \\
\hline
\end{tabular}

\section{3" Pompl a PALES ORIENTABLes :}

Angle de calage variant de $21^{\circ}$ à $6^{\circ}$ et caractérisée par les chifrres suivants à vitesse constante :

$\mathrm{H}=$ hauteur $(\mathrm{en} \mathrm{m}) ; \quad \mathrm{Q}=\operatorname{débit}(\mathrm{en} \mathrm{l} / \mathrm{s})$ $\mathrm{N}=$ puissance absorbée (en $\mathrm{kW}$ )

\begin{tabular}{|c|c|c|c|c|c|c|c|c|c|}
\hline $\mathrm{H}$ & 2 & 3 & 4 & 5 & 6 & 7 & 9 & 11 & 14 \\
\hline $\mathrm{Q} \mid$ & $390 \mid$ & 370 & 340 & 315 & 275 & 150 & 70 & 45 & 0 \\
\hline $\mathrm{N} \mid$ & 12,5 & 11 & 17 & 20 & 22,5 & $18^{\prime}$ & 19 & $19,5 \mid$ & 20 \\
\hline $\mathrm{B} \mid$ & $21^{\circ}$ & $21^{\circ}$ & $21^{\circ}$ & $21^{\circ}$ & $21^{\circ}$ & $15^{\circ}$ & $12^{\circ}$ & $9^{\circ}$ & $6^{\circ}$ \\
\hline
\end{tabular}

Soit, à hauteur de refoulement constante :

$H=$ hauteur $($ en $m) ; \quad Q=$ débit (en $\mathrm{l} / \mathrm{s})$ $\mathrm{N}=$ puissance absorbèe (en $\mathrm{kW}$ )

\begin{tabular}{|c|c|c|c|c|c|c|c|c|c|}
\hline $\mathrm{H}$ & 4 & 4 & 4 & 4 & 4 & 4 & 4 & 4 & 4 \\
\hline $\mathrm{Q}$ & 550 & 425 & 340 & 280 & 225 & 114 & 47 & 27 & 0 \\
\hline $\mathrm{N}$ & 35 & 21,5 & 17 & 14 & 12,2 & 7,8 & 5,6 & 4,3 & 3,1 \\
\hline
\end{tabular}

Après avoir déterminé pour chaque type de pompe les courbes puissance-vitesse, on obtient les courbes débit-vitesse de vent. L'allure de ces courbes au voisinage de l'amorçage est très différente suivant qu'il s'agit d'une pompe-hélice ou d'une pompe centrifuge. Le calcul du volume lotal pompé dans l'année et du nombre d'heures de fonctionnement donne les résultats suivants, loujours pour une puissance nominale établie a $8 \mathrm{~m} / \mathrm{s}$.

\section{Pompe centrifuge :}

Vitesse d'amorçage $\nu=6,75 \mathrm{~m} / \mathrm{s}$;

Volume d'eau annuel $\mathrm{V}=4.686 .000 \mathrm{~m}^{3}$;

Nombre d'heures de marche $=4.190 \mathrm{~h}$.

\section{Pompe à hélice :}

Vitesse d'amorçage $v=6 \mathrm{~m} / \mathrm{s}$;

Volume d'eau annuel $\mathrm{V}=4.953 .000 \mathrm{~m}^{3}$;

Nombre d'heures de marche $=5.250 \mathrm{~h}$.

\section{Pompe à pales orientables:}

Vilesse d'amorçage $v=4,5 \mathrm{~m} / \mathrm{s}$;

Volume d'eau annuel $\mathrm{V}=5.455 .500 \mathrm{~m}$;

Nombre d'heures de marche $=6.850 \mathrm{~h}$.

La pompe hélice à pales fixes permet d'accroìtre le volume pompé de $6 \%$ et le temps de marche de $25 \%$. La pompe à pales orientables accroit le volume poumpé de $17 \%$ et le temps de marche de $63 \%$ par rapport à la pompe centrifuge, dans les mêmes conditions.

Ces chiffres illustrent bien l'intérêt de l'emploi d'une pompe-hélice et surtout d'une pompe à pales orientables. Certes cette dernière entraîne une plus grande complication mécanique, mais, pour les machines d'une certaine puissance, le Gain de volume et surtout de temps est tel que cetle complication se justifie parfaitement. Si l'emploi de la ponıe à pales orientables reste pratiquement limité à des hauteurs de refoulement de l'ordre de 6 à $8 \mathrm{~m}$ au maximum, par contre la pompe-hélice peut être utilisée pour des hauleurs beaucoup plus grandes dans une disposition à plusieurs étages en série. 


\section{CONCLUSION}

Disons pour terminer qu'il existe actuellement des types d'éolienne parfaitement adaplés à l'entraìnement des pompes : soit éolienne lente pour pompe à piston, soit éolienne rapide pour pompe centrifuge ou hélice. Toutes permetlent, dans leur domaine particulier, d'obtenir une production hydraulique importante et un nombre d'heures de marche satisfaisant. Pour des régions moyennenrent ventées, ces groupes sont lout à fait rentables et peuvent concurrencer Lrès avantageusement les groupes de pompage électrique et thermique. Leur supériorité est évidente pour les points d'eau éloignés de centres habités ou de réseau de distribution. Si des déboires onl été enregistrés, ils doivent être imputés plus à un manque d'information sur la valeur des rents ou à une mauvaise adaptation du matériel (qu'à un rice propre au pompage par l'énergie du vent. Dans tous les cas, et principalement pour les groupes rapides d'une cerlaine importance, un équipenent convenable doit s'appuyer sur une prospection préalable du régime des vents et sur une élude sérieuse de l'adaptation des caractéristiques des machines utilisćes. Ces conditions ćtant remplies, l'éolienne résoudra économiquement de nombreux problèmes de pompage.

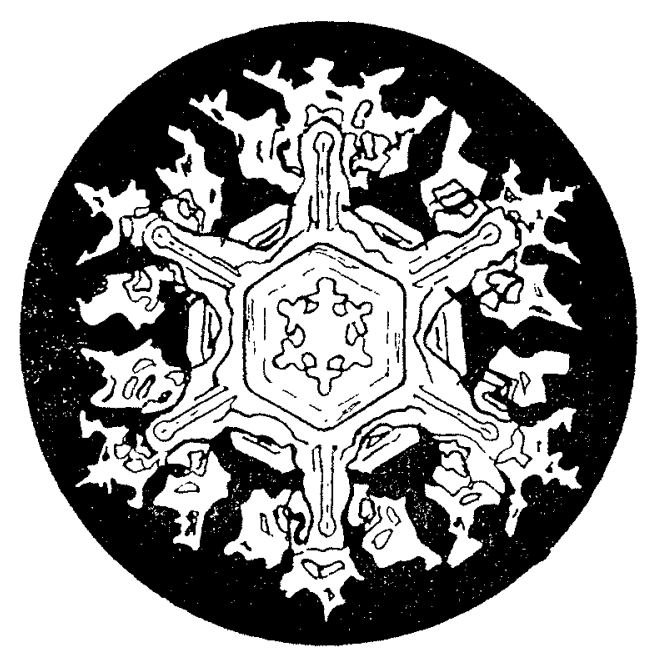




\title{
Water pumping by windmills
}

\author{
BI L. TADOT

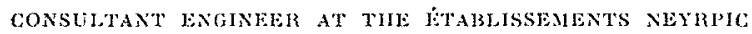

(Traduction by Madge E. Tmompson)

(See French text p. 496, for illustration)

\begin{abstract}
A brief historical review shows that, since remote antiquity, wind power has been used for pumping water. LEUPOLD and BELIDOR, and others, give some very interesting descriptions of pumping mills.

Modern machines include slow-running windmills for piston pumps and fast-running windmills with particularly suitable characteristics for driving centrifugal and propeller pumps.

The choice of the starting wind speed, rated speed and operating point of the pump have a
\end{abstract}

great infuence on the production and economy of the pumping set. Calculations, with supporting examples, for a slow-rinning windmill driving a piston pump, and for a high-speed windmill with a centrifugal pump, give some particularly interesting resulls, which are shown in the utilization graphs.

A comparison of the different types of pumps which may be coupled to a high-speed windmill shows the considerable advantage of using a pump with variable-pitch blades for low and medium lifts.

\section{HISTORICAL REVIEW}

To his many other claims to glory, Hammurabi, founder of the Babylonian empire, might have added that of introducing hydro-power into the realm of agriculture. We are told that it was he who, about 2000 B.C., according to some authorities, or 1700 B.C., according to others, conceived an immense project for using the energy of the wind for the irrigation of the plains of Mesopotamia. Whether this was only the ambitious desire of a sovereign, or whether the brave machines, like the celebrated hanging gardens, have disappeared for ever in the silt of the rivers, we do not know. But if Persia is generally considered as the cradle of the windmill, near-by Mesopotamia might well be country which first used the force of the wind for pumping water.

We have no information about these antique machines; at the most we can imagine that they were vertical-axis mills rather similar to those of which traces still remain on the Iranian plateaux. Traditionally they are held to have been introduced into Europe by the Crusaders. It is probably an over-simplification of the case to think that all the wonders of the mysterious East were brought back by gallant knights; nevertheless, many roads led to Palestine and at that time adventure began when the familiar horizon was passed. In any case, the windmill seems to have been used in Mediterranean countries well before the Crusades. From there it spread rather slowly to northern Europe; windmills are mentioned in the archives of Croyland Abbey in England from the year 1000. In France, ancient documents mention the construction of other mills about 1100. The first mills appeared in Holland in the thirteenth century and in Germany in the fourteenth century. In contrast with their Asiatic ancestor, with its vertical axis, the European mills all had a horizontal axis, generally with a four-bladed rotor in the form of a cross, which has made their silhouette familiar. Until the fourteenth century, the work of these machines was to grind corn, hence the name "mill" which still attaches to them.

About 1350, in the Low Countries, mills were first used for pumping water with the object of draining the land siluated below sea level. Gradually a suitable type of machine was developed for this new function and several, daling back to 
1430, were installed in the region of Schoonhaven in South Holland. Pumping mills did not take lons to spread in a country where they were to become a vital necessity. From Holland, where they werc lleveloped, they extended all over Europe, sometimes pumping water for the needs of agriculture, sometimes feeding the fountains adorning the park of a castle.

Descriptions of these machines are found in the works of engineers of the period. Ramelli, in "Le Diverse e Artificiose Machine", invites us to admire a mill of the classic type driving a double chain pump for feeding an ornamental fountain. The entire mechanism, which can be turned by means of a capstan, is mounted on a circular track situated at the top of a tower used as a water tower.

The chain pump was frequently used and is seen in the design of a machine proposed by Böckler in his Theatrum machinarum novum. It is a feature of Besson's curious machine of which the verticalaxis rotor recalls the modern Savonius rotors. It is found again in the engravings of Belidor's Archilecture Hydraulique.

The illustrations in the works of sixteenth and seventeenth centuries show evidence of an imagination inspired more by artistic vagary than by regard for construction. In the eighteenth century, on the other hand, engineering principles assert themselves; the machinery becomes rational, balanced and practical. The engineer is more in eridence than the artist in the drawings; numerous details show signs of the evolution which led to constructions so durable that they still survive in their main outlines.

The most interesting machine is undoubtedly that proposed by Jacob Leupold in his "Schauplalz der Wasser Künste" or "Theatrum machinarum hydraulicarum", printed in 1724 at Leipzig. An eightbladed rotor drives, by a crank shaft and tie rod, a single-acting piston pump, according to an arrangement adopted two centuries later for the socalled American windmills. Furthermore, Leupold's machine is even self-regulating. Each of the blades, which is able to pivot round an axis, is maintained by a spring system such that it is effaced progressively in a high wind and thus the rotor revolves no more rapidly in a gale than in a medium wind. The same work describes a number of mechanisms with crank shaft, tic-rod and slot for the control of piston pumps, which all show great ingenuity.

Belidor also was interester in machines for pumping water by means of the wind. In his Architecture Hydraulique is a description, among others, of a mill of which the axis, very much inclined, supports the wind wheel on one side and on the other an elevating wheel, a sort of scoop-type water-wheel, plunging directly into the feeding basin. Similar machines are still working in Friesland.

Belidor also gives a description of a mill driving a Persian wheel by means of a rope drive to reduce the speed of rotation; the sails are of an unusual shape for those days and foreshadow the high. speed propellers of today. It is unlikely, considering the means available at the time and the rudimentary construction of the sails, that such a machine could have worked satisfactorily. Nevertheless, one is struck by the modern appearance of the apparatus with its narrow and appropriately twisted blades.

Towards 1745 Edmund Lee patented the auxiliary wheel, the "fantail", which ensures the automalic turning of the mill into wind-an excellent device which is still used. This invention could also be attributed to Andrew Meikle, who later constructed slatted sails.

In 1759, Smeaton presented to the Royal Sociely a report "On the construction and effects of windmill sails" in which he describes his experimental researches with a device enabling torque mosurements to be made at different speeds on a model with a small number of sails. It can be said that with Smeaton the study of the windmill entereri into a scientific phase.

It was certainly in Holland that the windmill in its ancient form attained the highest degree of perfection; it is not an exaggeration to say that a large part of the country owes its existence to those marvellous machines. Marvellous they certinly were for the period. They were made entirely of wood, with their four sails in the form of a cross atlaining a span of nearly $20 \mathrm{~m}$ and driving, through an ingenious mechanism, elevating wheels, scoopwheels, or Archimedes' screws. From mill to mill, the water was thus raised from the polder fo the sea, solely by wind energy. About 1630 the lake of Schermeer, near Alkmar, in North llolland, was drained in this way according to the plans of Leeghwater. An area of about 11,710 acres was recovered and drained by a group of 50 mills driving back the water in two stages. The same engineer planned the draining of the sea of Haarlem with 160 windmills. Here were enierprises on the scale of the twenticth century! May we not conclude that the pumping windmil reached, in the seventeenth century, a stage of almost industrial development?

The very large number of mills used in Holland led to standardization, so that, in each region, they belonged to a few types only, repeated many times over: Wipmolen, perehed on its pyramidal base; Weidemolen, with its octagonal tower serving as a habitation; Binnenkruier, with its squat form. After centuries of good and loyal service, the windmill remains one of the characieristics of the buteh landscape.

\section{Miéteorological data}

The first requirement in planning a windmill pumping installation is a knowledge of the intensity of the winds in the place where it will be used. For a period of at least a year, measurements or betfer still records of the intensity of the wind have to be mate at the point considered. When, for economical reasons, it is not practicable to make a record of a complete vear, it is advisable to obtain typical recorls for the different periods of the year so that account can be taken of the seasonal influences on the wind regime. The measuring apparatus should be placed on a support 
of approximately the same height as the projected windmill. Meteorological readings are often made at the slandard height of $10 \mathrm{~m}$, but generally the axis of the rotor will he at a greater height-ol the order of $15 \mathrm{~m}$. It is preferable to adopt a height of $15 \mathrm{~m}$ for the measuring apparatus thus avoiding the necessity of estimating, from the results obtained at $10 \mathrm{~m}$, those which might have been obtained at $15 \mathrm{~m}$-a rather uncertain estimate in any case, as one does not generally know the local law of variation of wind intensity with altitude. Although it is generally agreed that the wind speed varies as $\mathrm{H}^{1 / 5}$, this is only an approximate law which may be considerably modified by the contours of the surrounding district. Also it is hardly valid if the measurements are being taken, as is often the case, in the layer of air directly disturbed by the neighbouring obstacles. It is scarcely necessary to add that records made too near the ground, or in the neighbourhood of objects such as buildings, trees, etc., ale useless.

Measuring instruments of the counter type are sometimes used, giving directly the total energy theoretically available per square metre of surface offered to the wind during the period which separates two consecutive readings. They have the advantage of being economic in operation, but they indicate only the total quantity of wind energy available without giving the fundamental detailswhich must be taken into account in determining the economic value of a project-of how that guantity is made up. Also wind energy counters should be used only for a broad general survey of a district. The ordinary direct reading anemometer is practically useless because of the large variations of wind intensity. The only instrument which can be recommended is the recording anemometer giving the variations of wind intensity as a function of time. All the information necessary can be obtained from these records. It is convenient, however, in order to facilitate the interpretation, to translate the readings into simple language relating to the determination of the following characteristics: velocity-duration curve, windfrecuency or power-duration curve, output characteristic of the pump, etc.

The velocity-duration curve indicates, for a given period and for each value of the wind speed, the number of hours during which the wind speed is equal to, or higher than, the speed considered. This curve is generally made for a year, but, in areas with a very marked seasonal regime, it is preferable to make it season by season. It is drawn from the anemometer reading; the corresponding analysis is rather laborious but can be greatly facilitated by the use of a special reading instrument.

Velocity-duration curves nearly all have the same shape. The part relating to the low wind speeds is generally unreliable as a result of the lack of sensitivity of the anemometers at wind speeds below about $2 \mathrm{~m} / \mathrm{s}$, but this part is of little importance from the point of view of energy. Velocityduration curves can, in the main, be characterized by the mean value of the wind for the period considered; this is called the "annual mean wind speed". This mean ligure obviously gives no indication of the regularity of the wind.

The wind-frequency curve, on the other hand, gives an idea of the regularity of the wind. It is derived quite simply from the velocity-duration curve and shows the number of hours, for the period considered, during which the wind speed remains within a given range; it therefore represents simply the variations of the inverse of the slope of the preceding curve. It always has a more or less pronounced maximum. Generally, the wind speed corresponding to the maximum of the frequency curve increases at the same time as the mean speed for the period considered. It may happen, in countries with a particular wind regime, that the frequency curve has two maxima.

The frequency curve which gives information on the wind regime is usefully complemented by the power-duration curve. As the power supplied by the wind is proportional to the cube of the speed, a curve giving, for each range of wind, a quantity proportional to the energy available can be obtained by multiplying the ordinates of the windfrequency curves by the cube of the speed. Thus again curves are obtained which present a maximum, but, while for the wind frequencies the absolute value of the maximum diminishes when the mean wind increases, the energy corresponding to the maximum increases when the mean wind increases.

- To facilitate the determination of the capacity to be installed, it is useful to trace the curves giving the energy produced for a given increment of wind speed, i.e., the increase of energy recoverable when the wind speed, for which the machine is designed, passes from $v$ to $v+\Delta v$. All these curves can be derived without trouble from the velocity-duration curve summarizing the information.

When the energy produced by varying winds cannot be directly used, as would be the case with a machine feeding its output into a high-capacity network, it is necessary to consider the possibilities of storing this energy, which is extrcmely variable with time, in order to adapt it to a regular utilization. The problem of storage arises for windmills generating electricity to be fed into an independent network; it also frequently arises for pumping windmills to give a water supply independent of any electricity supply network. There is the same problem again for drainage windmills, but in this case the reservoir will be at the suction instead of at the delivery.

The resolution of the problem of storage in a reservoir makes it necessary to determine the levelopment, in time, of the volume of water pumped by the machine and to compare it with the water requirements. In a simple case where the demand is constant, the optimum equipment, from the point of view of energy, would be such that the average rolume pumped in a given period, e.g., a year, by the windmill, would be equal to the quantity demanded. Because of the irregularities of pumping, it is necessary to provide a reservoir with a capacity depending on the variability of the winds. The variations of delivery of the pump can easily be represented on a grapl of delivery accumulated plotted against time; this is a curve which 
is continuously increasing and is more or less irregular. On such a graph, a constant delivery demand is represented by a straight line passing through the origin. The difference between the ordinates of the curve of windmill output and of the straight line of water consumed represents the function of the storage reservoir. In this way the curve is obtained representing the development in time. of the delivery supplied, or absorbed, by the reservoir, $Q_{r}$. The area situated under this curve represents the total volume of water supplied or absorbed:

$$
\mathrm{V}=\int_{0}^{t} \mathrm{Q} \cdot d t
$$

If the windmill is well adapted to the delivery required, the curve of the volume of the reservoir will oscillate more or less regularly about zero value. If the windmill is too strong for the demand, the curve for the reservoir will be diminishing, which means that the delivery supplied is greater than the demand; if, on the other hand, the windmill is inadequate, the curve of the volume of the reservoir will be continuously increasing, though obviously with a few irregularities.

When the windmill is suitably chosen, the capacity of the reservoir will be defined by the maximum value of the curve :

$$
\mathrm{V}=\int_{0}^{t} \mathrm{Q}_{r} d t
$$

It is obvious that, in practice, it is advisable to adapt the consumption to the seasonal irregularities of production, but the daily irregularities, or those spread over a few days, will be absorbed by the reservoir. It is necessary, therefore, to draw the curve representing the development in time of the quantity pumped; it will be derived from the anemometer recordings, using a curve giving, as : function of the wind speed, the quantity pumper for a given machine. This determination is laborious and would be greatly facilitated by the use of automatic analysing apparatus which would, in addition, enable the results to be obtained for several types of machines from the same recording.

\section{Apparatus \\ for analysing anemometer readings}

Before the anemometer readings can be used, they have to be translated into velocity-duration or velocity-frequency curves. The work of analysis can be done mechanically by means of a special apparatus, which consists of a device to unroll the recording paper, situated in front of a set of electrical contacts spaced out according to a scale of specds. $A$ sliding contact can be moved over these contacts and a pen, connected to the sliding contact, makes it possible, by following the curve by hand as it advances, to bring the sliding contact into touch with the contact corresponding to the speed indicated by the curve. Every time such a connection is made it closes the circuit of an impulse counter.
This is supplied by an impulse gencrator consisting simply of a battery and a toothed wheel forming a circuit-breaker with a speed of rolation proportional to the speed of movement of the paper.

Thus, all the counter elements corresponding to a speed equal to, or lower than, that indicated by the tracing pen advance by a number of units corresponding to the path followed on the paper. In the course of analysis, it is sufficient to read the figures given by the counters in order to obtain, at any instant, the state of the velocily-duration curve. It is also possible to complete this apparatus with a device enabling the output characteristic of the pump to be obtained for a given windmill. For this purpose, a second series of conlacts is placed beside the first. Each of the contacts is connected to a resistance of which the value is inversely proportional to the delivery from the windmill for the speed considered. The sliding contact connected to the tracing pen closes a circuit consisting of a current source and a counter. As the unrolling speed is constant, the counter indicates at each instant the volume of water pumped. It is, of course, possible to place on the same series of contacts another set of resistances supplying another counter and representing another machine. One can thus obtain, in the course of the same analysis, the pumping outputs by windmills of different characteristics.

\section{Utilization of meteorological data for determining the characteristics of the installation}

Being in possession of the different meteorological data, we can now see how to use them to define the performance of an installation. The operation of the windmill is linked up with lhree values of the wind speed :

(1) The speed $v_{1}$, called the "rut-in speed", is the speed at which the machine begins to supply power, i.e., in the case of a pumping installation, the speed at which the pump begins to deliver water. This speed must not be confused with the "starting speed", $v_{0}$, at which the machine begins to lurn but without supplying usable power. When a piston pump is being driven, $v_{0}$ and $v_{1}$ are confused, but with a centrifugal pump, $v_{0}$ and $v_{1}$ are distinctly different.

(2) The speed $b_{n}$, called the "rated speerl", is the speed at which the full oulpul of the maching is reached. Above this speed, the regulating device comes into operation to limit the output and keepl it constant.

(3) The speed $w_{\text {min }}$, caller the "furling speed", is the speed at and above which the machine is pul out of operation by a regulator or safety deviec. These three speeds define, on the velocity-duration or power-duration diagram, the zone of utilization. The choice of the speeds $v_{1}$ and $v_{n}$ is of the highest importance. The speed $v_{\text {and }}$ depends solely on the method of controlling the apparalus; it has litte influence on the energy produced as very strong 
winds are of short duration. If we consider only the total energy produced, the choice of the cut-in speed $v_{1}$ is not of great importance because the energy produced from low winds is always very small. On the other hand, for a machine operating independently and having a storage reservoir, the choice of this speed is very important, for it will finally determine the size of the reservoir to be provided.

\section{Choice of the cut-in speed}

Obviously there is an advantage in making this speed as low as possible in order to increase the number of hours of operation of the installation. Nevertheless, its choice is the result of a compromise between the cost of the reservoir and that of the machine. In fact, if the size of the reservoir diminishes when the cut-in speed is reduced, on the other hand, the diameter of the windmill increases when this speed diminishes, since, for a given installation, the torque absorbed by the pump at cut-in remains constant. The problem of the choice of the cut-in speed occurs in a different fashion according to whether the windmill drives a piston pump or a pump of the centrifugal kind.

In the first case, the problem concerns the starting forculu; it involves only the diameter of the windmill or the reduction ratio of the gearing, for the pump is of the constant-torque type.

The second case is more complex, for not only the dimensions and the speed of the windmill have to be determined, but also the operating point of the pump may vary according to the diameter of the rotor and its speed of rotation. It is no longer simply a question of obtaining a certain torque at zero speed, as for the piston pump, but of obtaining a torque at a certain speed, these two values depending on the characteristics of the centrifugal pump.

\section{Choice of the rated speed}

The choice of this speed is chiefly bound up with the total production of energy in the year. Two starting points may be envisaged: either the characteristies of the pump are considered (lift, delivery), or the diameter and type of windmill. If the efficiency of the windmill and of the pump remained constant at fractional loads, there would be an advantage in choosing a high value for $v_{n}$. But this is not generally the case. Taking into account the variations of efficiency of the windmill and of the pump, one will try to obtain the maximum volume of water in the year for a given windmill, or, on the other hand, the smallest windmill for a given volume of water.

When driving a centrifugal pump, the choice of the rated speed camnot be made independently of the choice of the cut-in speed, the ratio of these speeds being laid down for a given pump. In fact, with a given lift, the difference in speed of rotation of the pump between running at maximum efficiency and running at zero delivery, or at cut-in, is small.
This difference can be increased only by choosing an operating point considerably distant form the point of maximum efficiency. It is therefore necessary, for a given pump, to try to move the points $v_{1}$ and $v_{u}$ on the velocity-duration curve so as to effect the best compromise between the total volume of "water pumped and the volume of the reservoir necessary to take into account calm periods, or else to choose a pump which makes it possible to have a suilable ratio between $v_{1}$ and $v_{n}$. One cannot, however, adopt too high a value for this ralio without moving away from the zones of reasonable efficiency. The difficulty that exists in obtaining a suitable value for the ratio $v_{n} / v_{1}$ could be surmounted to a certain extent by making the gear ratio between windmill and pump vary. But this would involve expense, because the variation of this ratio would have to be automatic if it were to come into operation at each change of wind speed; an arrangement such as this does not seem to have been put into practice up to the present.

\section{Equipment normally used}

Two types of pumping set are available to the user : slow-running windmills with piston pumps, of which there are at present a very large number, and high speed windmills coupled to propeller pumps or to centrilugal pumps. These last machines, of recent construction, are a modern develoj)ment.

\section{(a) LOW-POWER INSTALLATION WITH PISTON PUMP :}

At first sight it does not scem obvious, and strictly speaking it is not essential, that a low-power pumping installation should be equipped with a piston pump. But, on the one hand, the height of the lifts, and, on the other hand, the need for simple equipment, generally lead, for low powers, to the use of slow-running piston pumps.

The windmill to use in this case is one of low specific speed, nearly always, with a reducing gear. This kind of machine has a high starting torque, which is perfectly in keeping with the requirements of piston pumps. As rotors of low specific speed have a large number of blades, the use of only fixed blades can be envisaged; the method of regulation which is then required to limit the speed, the power and the stresses in a high wind is by spilling. The kind of apparatus which is thus called for cannot reasonably be constructed beyond a eertain dimension. Because of the inertia of rotors with a large number of blades, the behaviour of such a machine in gusts prohibits its use for large (liameters. As an indication, a diameter of 7 to $8 \mathrm{~m}$ is the maximum.

Then again, as the piston pump is palpably a pump with constant torque, the power of the windmill is badly used in a high wind. For small machines, this bad utilization is not serious, but, for large machines, where efficiency is more important, it would not be permissible. An attempt has been made to remedy it by devices modifying the stroke 
of the pump as a function of the wind speed, but nothing of practical use has resulted.

\section{(b) MediuM OR High-POWER INSTALLATION WITH ROTARY PUMP :}

The disadvantages just mentioned condemn the use of piston pumps for high-power pumping unless, of course, the height of the lift demands it, and then special arrangements are necessary. We are therefore led, according to the deliveries and lifts, to use centrifugal or propeller pumps. These pumps must rotate at high speed, hence the use of windmills of high specific speed, generally with gearing. The low starting torque of the machines is not a serious disadvantage, since the starting torque of the rotary pumps is also low. Naturally speed regulation is not necessary; it is only the problem of behaviour in a high wind that has to be solved. Such sets permit a good utilization of wind energy, but they have the disadvantage of providing only a low lift at starting. Although low winds account for only a small part of the annual production of energy, this it not a serious drawback. These sets are therefore quite suitable for districts where the winds are fairly constant.

For a low lift it would be advantageous to use a combination of the high-speed windmill with a propeller pump having blades of automaticallyrariable pitch, this pitch diminishing in a low wind to reduce the power absorbed. Such an arrangement would enable the cut-in in a low wind to be allied with obtaining a large delivery in a strong wind, the efficiency of the set always remaining near the maximum. This solution is obviously rather complicated from the mechanical point of view, but it mut be considered seriously for sets of a certain power at low lift.

Finally, for high powers, electrical transmission between the windmill and the pump may be necessary; one must not lose sight of the fact that the most favourable places for wind energy are seldom those where there are springs or wells. Electrical transmission has the great advantage of avoiding all the difficulties of mechanical connection, which involve great expense for high powers; it has the further advantage of permitting the direct use of electrical energy, should the occasion arise.

\section{Slow-running windmills for piston pumps}

These machines have a rotor with a large number (from 16 to 24 ) of fixed blades made of sheet metal and not profiled. This rotor drives, through a speed-reducing gear, a mechanism which converts rotational motion into a reciprocating motion by means of rods, slots, eccentrics, etc. All these mechanisms are nearly equivalent, the efficiency depending more on the cuality of the performance than on the principle used. Speed reduction between the rotor and the mechanism driving the pump is necessary for great depths of pumping to reduce, on the one hand, the inerlia stresses, and to avoid, on the other hand, the resonance phenomena in the pump-rod, which, as a result of its length, behaves like a tension spring. As this equipment has to be mass-produced, speed reduction is retained even for small depths of pumping. Generally the same mechanism enables two values for the stroke of the pump to be obtained by simple replacing of the crank-pin. The regulation of the power in a high wind is always effected by spilling, the rotor turning out of wind when there is a gale. For this purpose, the tail vane is articulated and joined to the hody of the machine by a spring; the spilling moment is obtained, either by a side vane, or, much more often, by placing the axis of the rotor slightly off centre in relation to the yawing axis. I'his method of regulation is simple and effective. It has, however, the disadvantage of producing shocks with sudden variations of wind speed, the machine, under the action of the spring, returning violently to its position of operation facing the wind. This is remedied very efrectively by the use of a shock-absorber, of the automobile type, mounted on the joint of the tail vane; such a shock-absorber is particularly recommended for machines of large diameter.

Different types of pumps can be driven by the slow-rumning windmill. In view of the method of working by a rod rescending the whole length of the lift pipe, which is therefore best applied to handling tensile loads, the single-acting pump is the most suitable and much more largely used. It has the disadvantage in operation, however, of presenting great irregularities in the torque absorbed, since it demands power only during a half-turn of the crank shaft, the descent of the piston being made under its own weight.

The reduction of this irregularity would be of advantage because it has a direct effect on the starting torque and therefore, finally, on the length of time operation of the machine. One may try to reduce it by the use of a double-acting pump, but the driving rod of the piston will be allernately submitted to tensile and compressive loads; guiding of the rod is necessary to avoid buckling and this method is rarely used.

Another possibility would be to use a pump with two pistons in the same body, with two driving rods; this is very interesting from the point of view of the regularity of the torque absorbed, as well as, naturally, of the regularity of the delivery, but the driving mechanism is much more complicated and that is why it is not used. This arrangement could perhaps be considered in cases where the lift pipes are long.

As the single-acting pump is adopted for its robustness, attempts are made to even out the torque which it absorbs by an external device such as a lever, weight or spring. The spring system seems to be the best, because in avoiding additional articulations it refuces the friction losses in the mechanism. The use of a compensating system has two favourable effects. It enables the machine to start at a lower wind speed and it reduces the stresses in the driving mechanism. The irregularity of tha torcue absorbed by the pump produces cyclic variations in the speed of the machine. In a single-acling pump without compensation, the rotor supplies power at the upstroke of the rod and slows down. On the other hand, at the down- 
stroke, the weight of the moving parts provides a torque. The rotor, no longer producing power, accelerates and accumulates kinetic energy which is restored at the following stroke. The variations in spoed of rotation which result are nevertheless quite small because of the inertia of the rotor and this irregular operation has no harmful effect.

To reduce the starting effort and to avoid starting with the full load on the piston, a permanent leak is sometimes made at the delivery valve. This arrangement has advantages and disadvantages, as will be seen.

The permanent leak establishes a communication between the two faces of the piston; diagrammatically the pump can then be represented by the sketch of fig. 16. If $Q$ is the deivery of the pump (volume swept out by the motion of the piston), $q$ the delivery passing through the leak and $Q_{e}$ the effective delivery passing up the delivery pipe, we have:

$$
\mathrm{Q}=q+\mathrm{Q}_{e}=\frac{\pi d^{2}}{4} \mathrm{~S} \cdot \frac{n}{60}
$$

when $d=$ diameter of the body of the pump;

$$
\begin{aligned}
& S=\text { stroke; } \\
& n=\text { number of strokes per minute. }
\end{aligned}
$$

If $\omega$ is the area of the leakage path and $H$ the pressure developed by the pump, we have:

$$
q=\omega \sqrt{2 g \mathrm{H}}
$$

If $\mathrm{H}_{0}$ is the lift head in normal operation (a value which is practically constant except for variations of level in the reservoir), the effective delivery will be zero as long as $\mathrm{H}$ is smaller than $\mathrm{H}_{0}$; then when the value of $\mathrm{H}_{0}$ is reached, the delivery from the leak will remain constant. The variations of the delivery and of the lift head can be represented on a graph as a function of the number of strokes per minute. The delivery $Q$ varies linearly. The head $H$ varies according to a parabola until the head $H_{0}$ is reached; it then remains constant. The head $\mathrm{H}_{0}$ will be reached for a number of strokes per minute at which the delivery from the leak will remain constant with the value $q_{0}$; it is only when starting from $n_{0}$ that the pump will deliver effectively. The area of the leakage path depends on the number $n_{0}$ adopted according to the equation :

$$
\omega=\frac{\pi d^{\dot{2}}}{4} \mathrm{~S} \cdot \frac{n_{0}}{60} \frac{1}{\sqrt{2} g \mathrm{H}_{0}}
$$

But because of the permanent leak, the efficiency of the pump will be reduced. Apart from the other inevitable losses (because of valves, pressure drops, etc.) the efficiency will be :

$$
r_{1}=\frac{\mathrm{Q}_{0}}{\mathrm{Q}}=\frac{\mathrm{Q}_{e}}{\mathrm{Q}_{e}+q_{0}}
$$

It will therefore be zero for $n=n_{0}$ and will then increase according to the above equation. This loss of efficiency is far from negligible, if we do not wish to risk using a cross section for the leak which is so ridiculously small that it may be blocked by a small grain of sand. If the leak becomes blocked, this is not a very serious matter, but it is more serious if the leak is enlarged by crosion (a current of water at high speed is continuously passing through it), for the efficiency then falls very rapidly.

A numerical example will show the difficulty better.

Take the case of a pump with the following characteristics :

$$
\mathrm{H}_{0}=50 \mathrm{~m} ; \quad d=120 \mathrm{~mm} ; \quad \mathrm{S}=385 \mathrm{~mm}
$$

If $n_{0}=3$, the leakage path will have to have an area of $0.072 \mathrm{~cm}^{2}$, and will be a circular orifice of diameter only $3 \mathrm{~mm}$.

If the speed of normal operation is $n=15$, the efficiency, because of the leak, will be :

$$
r_{i}=-\frac{15-3}{15}=80 \%
$$

Therefore, in normal operation, $20 \%$ of the power of the machine will be absorbed by the permanent leak. Certainly the machine starts at a lower wind speed than it would if there were no leak, but, all things considered, the gain is rather illusory. The fact that the machine starts is of little importance if it does not pump any water.

\section{Determination of the operating conditions of a slow-running windmill with a piston pump}

The windmill is distinguished by its characteristics giving the torque or the power as a function of the speed of rotation for a given wind speed and diameter. It is also interesting, in order to determine the strength of the parts and principally of the tower, to know the axial thrust as a function of the wind speed; these values are generally given in the form of non-dimensional coefficients.

Taking into account the friction losses in the driving mechanism of the pump and in the piston pump itself (due to friction, leaks, pressure drops), a practical power coefficient, or ratio of the hydraulic power output to the total power in the wind, has an average value of 0.2 . This rather low efficiency is made up in the following way :

Theoretical efficiency of a perfect windmill ...................

True efficiency of a slow-running rotor as a fraction of the theoretical effi-

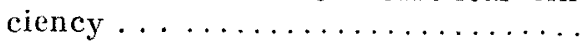

Efficiency of the mechanism - transmission, pump, pressure drops.......

It is useful to remember that the efficiency is a secondary consideration; what it is important to know is the cost per unit volume of pumped water. Taking a concrete exemple, let us determine the output of a slow-running windmill with a piston 
pump and then the cost per cubic metre of pumped water. The diameter of the windmill is $6.10 \mathrm{~m}$ and it has to pump water with a total lift of $40 \mathrm{~m}$. As the piston pump has a constant torque (except for the irregularities mentioned above, but in normal operation, and taking into account the inertia of the rotor, everything takes place as if the machine absorbed a constant torque), it is convenient to represent the characteristic of the windmill as a graph of torque plotted against speed with the wind speed as parameter. It is necessary first to choose the bore and the stroke of the pump. Consider four models of pumps resulting from the combination of two pumping installations with two crank positions :

\begin{tabular}{|c|c|}
\hline $\begin{array}{l}\text { bore } \ldots \ldots \\
\text { stroke } \ldots \ldots \\
\text { capacity of cylinder.... }\end{array}$ & $\begin{array}{l}125 \mathrm{~mm} \\
384 \mathrm{~mm} \\
4.7 \text { litres }\end{array}$ \\
\hline $\begin{array}{l}\text { bore } \ldots \ldots \\
\text { stroke } \ldots \\
\text { capacity of cylinder. }\end{array}$ & $\begin{array}{l}125 \mathrm{~mm} \\
490 \mathrm{~mm} \\
6 \text { litres }\end{array}$ \\
\hline $\begin{array}{l}\text { bore } \ldots \ldots \ldots \ldots \\
\text { stroke } \ldots \ldots \ldots \ldots \ldots \\
\text { capacity of cylinder.... }\end{array}$ & $\begin{array}{l}160 \mathrm{~mm} \\
384 \mathrm{~mm} \\
7.7 \text { litres }\end{array}$ \\
\hline $\begin{array}{l}\text { ore. } \\
\text { roke. }\end{array}$ & $\begin{array}{l}160 \mathrm{~mm} \\
490 \mathrm{~mm} \\
9.9 \text { litr }\end{array}$ \\
\hline
\end{tabular}

The intersection of the horizontal line of constant torque of the pump, with the torque curves at constant wind speed of the windmill, enables the speed of rotation of the machine as a function of the wind speed to be determined for the four models of pumps. From this the curves giving the delivery as a function of the wind speed are easily obtained. It will be noticed that the envelope of the four curves is a cubic curve corresponding to the power co-efficient of 0.2 . The zones of tangency to the envelope are the zones of higher efficiency.

It will be seen therefore that the choice of the type of pump depends on the wind speed for which it is to be designed. The machine is not called upon to function for only one value of wind speed but for a whole range of speeds, with durations characterized by the velocity-duration curve of the district considered. Starting from the velocityduration curve and the delivery curves as a function of the wind speed, it is easy to determine for each type of pump the total volume of water pumped in the year as well as the total number of hours of operation. Account can also be taken of the wind speed at which wind spilling by the rotor begins to come into operation; at and above this speed, the power, and therefore the delivery, of the machine is kept constant. The results obtained, starting from the velocity-duration curves corresponding to the annual mean wind speeds $(6 \mathrm{~m} / \mathrm{s}$ and $4 \mathrm{~m} / \mathrm{s})$, are shown on a graph of annual delivery plotted against number of hours, i.e., a utilisation graph. It can thus be seen that, with an average wind of $4 \mathrm{~m} / \mathrm{s}$, il is possible to pump up to $30,000 \mathrm{~m}^{3}$ per year, with a lift of $40 \mathrm{~m}$, the period of operation being 6,000 hours, i.e., for $68 \%$ of the time. On the other hand, with an annual average wind of $6 \mathrm{~m} / \mathrm{s}, 50,000 \mathrm{~m}^{3}$ per year will be obtained, with a period of operation of 7,500 hours, i.e., $85 \%$ of the time.

Finally, by a reduction of the period of operation, it will be possible to increase the delivery pumped up to $72,000 \mathrm{~m}^{3}$ per year. In view of the advantage that there is, for this type of installation, in obtaining the maximum time of operation, consider only the point corresponding to $50,000 \mathrm{~m}^{3}$ and 7,500 hours. The pumping of $50,000 \mathrm{~m}^{3}$ per year at $40 \mathrm{~m}$ represenis a quantity of hydraulic encrgy equivalent to $5,500 \mathrm{kWh}$ per year. If the windmill were replaced by an electrical pumping set, for which it would be necessary to allow a total efficiency of 0.53 , i.e. : efficiency of the motor............. 0.80 loss on line.. .................. 0.05 total efficiency of the pump, including trans-

mission . . ............... 0,70 there would be a consumption on the meter of $10,400 \mathrm{kWh}$ per year. For pumping by an internal combustion engine there would be, under the same conditions, an annual consumption of 4,100 litres of fucl oil.

Without taking into account the costs of installing the supply line for the electrical apparatus, nor the costs of supervision and maintenance for the thermal set, such a windmill installation could be written off in less than seven years, while one can count on a normal life of twenty years with suitable maintenance. As the costs occasioned by the line and by the necessity for supervision of the thermal engine become considerable when the pumping set is situated at some distance from an inhabited centre, the economy of the windmill is considerably increased under these conditions so that it may become much more advantageous.

\section{High-speed windmills for centrifugal or propeller pumps}

A "high-speed" windmill is a machine with a small number of blades of which the speed of rotation corresponds to a tip-speed ratio $n / v$ of the order of 3 to 7 or 8 and even more. These machines have very different operating characteristics from those of the slow-running windmills. The variation of the torque coefficient as a function of the ratio $a / v$ is as follows:

At starting, the torque is very low, then increases with the ratio $u / v$ until it reaches a maximum which is situated approximately in the zone of maximum efficiency. After this maximum, the torque decreases continuously while the ratio $u / v$ increases up to the point when the machine races. The zone of increasing torque between starting and the maxinum often corresponds to instabilities of operation. The very low starting torque of these machines makes them unsuitable for the direct drive of piston pumps, which can be suitably driven only if, between the windmill and the pump, either an electrical transmission, or a centrifugal cluteh, enables the windmill to acquire speed in order to reach an operating condition with sufficient torque. 
On the other hand, centrifugal or propeller pumps are very well adapted for coupling to such windmills; their starting torque is very low and their speed-power characteristic is such that it is possible to make the zones of operation at maximum efficiency of the windmill and of the pump coincide. It is therefore not surprising that with these sets a hydraulic production is obtained which is always appreciably higher than with piston pump sets for the same diameter of rotor and the same site. Certainly it is theoretically possible to obtain a good utilization of the slow-running windmill and a good production of energy with the piston pump sets, but this would involve mechanical complications, such as automatic variation of the pumpwindmill ratio, complications which do not accord with the robustness demanded of these low-power machines. It therefore seems desirable, for reasons of economy and operation, that the utilization of the slow-running windmill should be confined to low powers. While the high-speed windmill should have its application extended to medium and high powers, for which a good overall efficiency is necessary.

High-speed windmills may be of two types: either with fixed blades or with variable-pitch blades. The fixed-bladed machine uses a remarkable property of thin profiles for its regulation. When the angle of incidence of the relative wind with respect to the blade reaches a certain value, the lift coefficient of the profile decreases abruptly and the power of the machine is limited instead of increasing with the cube of the wind speed. This phenomenon, which is well known in aviation by the name of stall, can be used to limit the power of the windmill; it necessitates, however, the use of very thin blades, which may not always behave well in gales and in violent gusts.

The machine with variable-pitch blades allows greater flexibility of operation and fortunately solves the problem of behaviour in a high wind. The operation of this machine is as follows:

For wind speeds between the starting speed and the rated speed, the pitch of the blades remains constant; the machine behaves like a fixed-bladed machine the running of which is defined by the intersection of the windmill and pump characteristics. When the rated speed is exceeded, the regulating mechanism comes into operation to modify the pitch of the blades and to maintain the power and the speed constant.

Different methods may be used to control the pitch of the blades-centrifugal force, aerodynamic thrust on the blades, etc., or even, for high-power machines, servo-motors and governors. Regulation, using a spring system balancing the aerodynamic thrust of the blades, has the great advantage of behaving remarkably well in gusts, for under the effect of a sudden increase of wind speed the blades yield, limiting at the same time the speed of rotation and the loading to which the whole of the machine is subjected. Figure 21 shows such a machine coupled to a propeller pump. In this machine, the position of the pitch-change axis of the blades is chosen so that the resultant of the aerodynamic loads on the blade creates a torque which is always in the same sense; this torque, increasing with the wind speed, is balanced by a flexible system such that the speed of rotation is held approximately constant in the zone of regulation and tends to diminish in very high winds, the blades then feathering. As the moment of inertia of the blade around its pitch-change axis is very small, the flexible system yields immediately under the effect of a gust and the loading sustained by the whole construction is reduced. The value of the wind speed at which regulation begins, as well as that at which the blades feather, may be adjusted by regulating the initial tension of the spring. The shaft of the rotor drives a vertical shaft, co-axial with the tower, though a speedincreasing level gear. This shaft, extending to ground level, is connected to a pump with a vertical axis, an arrangement which is also suitable for submerged operation. The yawing of the entire machine round a vertical axis is effected by an auxiliary rotor which drives, through reduction gearing, an irreversible worm gear. This very effective device, besides being self-correcting, also limits the speed of yawing, thus avoiding excessive gyroscopic loads.

\section{Determination of the operating conditions of a high-speed windmill with a centrifugal pump}

Two sets of operating conditions must be considered for the windmill. When it is rumning with a wind speed higher than the rated speed and the regulating device intervenes, the output is kept practically constant and the set works under the same conditions as a normal pumping set with an electric motor. On the other hand. with wind speeds lower than the rated speed, the regulating device does not intervene and the machine runs like a fixed-bladed windmill. It is this mode of operation that we are going to consider particularly.

Whereas, in the case of a windmill operating with a piston pump, it is convenient to represent the characteristics of the windmill on a graph of torque plotted against speed of rotation, because the piston pump is a machine with constant torque, here, in view of the way in which the characteristics of the pump are usually given, it is preferable to represent the operating conditions of the windmill on a graph of power plotted against speed of rotation. On such a graph, and for a given wind speed, the variation of the power available on the shaft of the windmill, as a function of the speed of rotation, is represented by a curve with a single maximum. The part on the left of this curve is not generally used and corresponds to instabilities of operation, especially for high-speed machines. The apex of the curve corresponds to the zone of maximum efficiency; the machine races at a speed of rotation which is practically double that which occurs at the maximum efficiency condition. It is possible to proceed with fair accuracy from a curve corresponding to a value $v_{1}$ of the wind speed to a curve corresponding to the value $v$, by multiplying the abscissae by the ratio $v_{2} / v_{1}$ and the 
ordinates by the ratio $\left(0, v_{0} / v_{1}\right)^{3}$. The points of maximum efficiency are therefore approximately situated on a cubic curve $\mathrm{N}=k_{n}{ }^{3}$. This property also belongs, as it does to all turbine machines, to centrilugal and propeller pumps; thus one may expect that the zones of operation at good efficiency of the windmill and of the pump will happily coincide.

The characteristics of pumps are usually given in the form of a curve showing delivery as a function of lift, at a constant specd of rotation. To study the coupling of a pump to a windmill, it is more convenient to use the characteristics of power absorbed as a function of speed of rotation at constant lift, the latter being, in fact, practically constant in most cases. If, however, the pressure drop of the circuit on which the pump delivers were not insignificant, it would be convenient to use the curve of power as a function of speed of rolation corresponding to the delivery-lift characteristic of the circuit of utilization. It is easy to pass from the delivery-lift characteristics at constant speed to the power-speed of rotation characteristics at constant lift by the standard laws of similarity, either for the same rotor or for geometrically similar rotors. It is also convenient to plot the power-speed curves at constant lift for a range of deliveries. By superposing on the plot of power-speed of rotation the pump and windmill characteristics, intersections defining the points of operation may be obtained and thus will be defined, for each wind speed, the speed of rotation and the delivery supplied by the pump with the lift considered.

To determine the power-speed curve at constant lift for a given type of pump, it is necessary to know first of all the operating point at the rated condition. On the windmill, this point will be defined by the wind speed and the speed of rotation. Generally the point of maximum efficiency on the curve $v_{n}=$ Cte is chosen. This point being chosen. we know the power available and the speed of rotation. It remains to determine the pump. The type of machine being known and characterized, for example by a curve Q-H for $n=$ Cte for a machine of diameter $D_{1}$, we shall choose on the curve an operating point $\left(Q_{1}, H_{1}, n_{1}, N_{1}\right)$. We shall then find, by the standard laws of similarity, the diameter and the speed of rotation that it is necessary to give to this pump to make it absorb the power $\mathrm{N}_{2}$ for the lift $\mathrm{H}_{2}$.

Suppose $\mathrm{A}=\mathrm{H}_{2} / \mathrm{H}_{1}$ and $\mathrm{B}=\mathrm{Q}_{2} / \mathrm{Q}_{1}$.

We have $N_{2} / N_{1}=A$. B. Hence the ratio $B$ defining the delivery of the pump. The diameter and the speed are therefore given by :

and :

$$
\frac{D_{2}}{D_{1}}=\frac{B^{1 / 2}}{A^{1 / 4}}
$$

$$
\frac{N_{.2}}{N_{1}}=\frac{A^{3 / 4}}{B^{1 / 2}}
$$

A knowledge of $n_{2}$ enables, among other things, the gear ratio to be provided between the propeller and the pump to be defined. The pump is then determined by the values $D_{2,}, n_{2}, Q_{2}$. The curve $N-n$ for $\mathrm{H}_{2}=$ Cte required is no less than a transformation to the horizontal line $\mathrm{H}_{1}=$ Cite of the hump of the curve of the pump of diameter $D_{1}$.

The choice of the point defining the operation of the pump is obviously arbitrary. For sets with motor drive at constant speed, we shall choose a point in the region of best efficiency of the pump.

For a pump driven by a windmill, it is not at all obvious a priori that the point of maximum efficiency of the pump is the most favourable. It is necessary, in fact, to consider here that, on the one hand, the total production of energy depends on the velocity-duration curve of the site considered and that, on the other hand, the total number of hours of operation is of great importance for the calculation of the dimensions of the storage reservoir.

\section{Influence of the choice \\ of the operating points of the windmill and of the pump on the output}

To show the considerable influence of the choice of the operating point of the pump on the output of the set, we will take a concrete example, that of the coupling of a high-speed windmill of $13 \mathrm{~m}$ diameter, of which the power-speed characteristics are given in Figure 22, to a centrifugal pump of which the delivery-lift characteristics are given in Figure 23, the lift being $40 \mathrm{~m}$ and the meteorological conditions being defined by the velocilyduration curve in Figure 7 corresponding to an average wind speed of $6 \mathrm{~m} / \mathrm{s}$. The operating conditions are defined by the rated point of the windmill and the corresponding operating point of the pump.

For the windmill, the following point will bo considered :

operation at maximum efficiency for wind speed: of 6,78 and $9 \mathrm{~m} / \mathrm{s}$ (above these speeds the regulation keeps the power constani);

and for the pump:

the points marked A, B, C, D, E, F on the diagram and corresponding respectively to the values of efficiency of $72 \%, 80 \%, 73 \%, 66 \%, 5 \%$ and $45 \%$.

In all, 24 different sets of operating conditions will be considered. For each of them, as has been said above, a power-speed of rolation curve will be drawn up for $\mathrm{H}=40 \mathrm{~m}$ and the corresponding curve giving the delivery as a function of the wind speed.

Fig. 24 represents the delivery-wind spoed curves for the different operating points of the pump and for the rated wind speed of $8 \mathrm{~m} / \mathrm{s}$. It will be seen, among other things, that the cut-in of the pump occurs for a delivery which is no zero; this is che to the fact that the delivery-lift curve at constant speed has a maximum. If this curve did not have a maximum, the cut-in would oceur at zero delivery, as will be seen for the other types of pumps considered later. 
The velocity-duration curve defining the meteorological regime gives, for each wind speed, the number of hours of operation and enables us to calculate, from the delivery-wind speed curve, the total volume of water pumped and the total numbers of hours of operation, for a year.

Each of the 24 sets of operating conditions envisaged thus leads to a group of figures summarizing the annual output. These results are given on a graph of total delivery plotted against number of hours; the different points are grouped according to curves of equal rated wind specds and of equal conditions of operation of the pump. The graph thus obtained clearly shows the considerable influence of the choice of the opcrating points on the output. I't can be seen, in particular, that the operating conditions corresponding to the maximum efficiency of the pump at the rated point are far from giving the best results; it is necessary, on the contrary, to select an operating point for the pump, at the rated operating condition, situated well to the right of the point of maximum efficiency. Thus, in the case considered, the maximum volume pumped is obtained for a rated efficiency of the pump of $66 \%$ and the maximum duration of operation for a pump efficiency at the rated point of only $45 \%$. The serious study of a windmill pumping project must include the tracing of such curves because they alone make it possible to choose correctly the characteristics of the installation to take into account both the requirements in total rolume and in number of hours of operation.

From the figures which have just been obtained, we can form an idea of the economy of the installation. Consider the operating point characterized by an annual output of $550,000 \mathrm{~m}^{3}$ at $40 \mathrm{~m}$ and 5,750 hours of operation. If the pumping were done by a pumping set with an electric motor, and allowing the following average efficiencies,

loss on line............... $5 \%$ efficiency of the motor......... $90 \%$

efficiency transmission and pump.. $70 \%$

there would be an annual consumption of 107,000 $\mathrm{kWh}$. If the pumping were done by a thermal set, the annual consumption of fuel oil would be 34,000 litres. Under these conditions, and without taking into account the costs of installing the supply line, on the one hand, and the costs of maintenance and supervision, on the other hand (costs which may be high if the pump is situated at some distance from an inhabited centre), the windmill can be written off in lour years. The installation of a pumping set with a windmill is therefore particularly interesting. These conditions, which are already very favourable, may be still further improved by the use of other types of pumps, as we shall see.

\section{Influence of the type of pump on the output}

An essential measure of the value of a windmilldriven pumping set is the number of hours of operation. $\boldsymbol{T}_{\mathrm{C}}$ increase this number of hours, we have just seen that there is an advantage in choosing a rated operating point of the pump situated well to the right of the points usually chosen for electric motor or thermal sets. This is due to the fact that the delivery-lift characteristic of a centrifugal pump is very flat on the left side between zero delivery and maximum efficiency. It would be an advantage to be able to use pumps of which the delivery-lift characteristic was rising, even at low values of delivery. This is the case with pumps of high specific speed and particularly with propeller pumps. While, for a centrifugal pump, the lift at zero delivery is of the order of 1.2 times the lift at maximum efficiency on a curve at constant speed, on the other hand, for a propeller pump, it is not unusual to obtain a lift at zero delivery which is double that of the maximum efficiency.

The propeller pump therefore appears to be better adapted than the centrifugal pump to be driven by a windmill. Nevertheless, the gain on the number of hours of operation is less than might be expected, for the power absorbed at constant speed increases when the delivery diminishes. This difficulty can be overcome by the use of a pump with variablepitch blades which can be regulated in such a way that the pitch of the blades diminishes for low wind speeds; as the power absorbed, other things being equal, decreases when the pitch of the blades diminishes, this fault of the propeller pump may be corrected and the number of hours of operation increased.

A concrete example will show the particular features of the three types of pump. Consider a windmill of $13 \mathrm{~m}$ diameter possessing the characteristics shown on Fig. 22 and driving a pump delivering with a lift of $4 \mathrm{~m}$. The rated point is chosen for a wind speed of $8 \mathrm{~m} / \mathrm{s}$ and the velocity-duration curve corresponds to an annual mean wind speed of $6 \mathrm{~m} / \mathrm{s}$. The following pumps will be considered :

\section{(1) Centrifugal pump :}

deduced by similarity from the pump previously considered and used at the rated point with $n=80 \%$.

\section{(2) Propeller íum :}

(blade pitch angle $B=21^{\prime \prime}$ ) and characterized by the following figures for operation at constant speed :

$$
\begin{gathered}
\mathrm{H}=\text { Lift (in } \mathrm{m}) ; \quad Q=\text { Delivery (in } \mathrm{l} / \mathrm{s}) ; \\
\mathrm{N}=\text { Power absorbed (in } \mathrm{kW} \text { ) }
\end{gathered}
$$

\begin{tabular}{|c|c|c|c|c|c|c|c|c|c|c|}
\hline $\mathrm{H}$ & 2 & 3 & 4 & 5 & 6 & 7 & 8 & 10 & 12 & 14 \\
\hline $\mathrm{Q}$ & 390 & 370 & $340 \mid$ & 315 & 275 & 150 & 120 & 80 & 40 & 0 \\
\hline $\mathrm{N}$ & 12.5 & 14 & 17 & 20 & 22.5 & 25 & 27 & 32 & 37 & 42 \\
\hline
\end{tabular}

Or, for operation at constant lift.

$$
\begin{gathered}
\mathrm{H}=\mathrm{Lift} \text { (in } \mathrm{m}) ; \quad \mathrm{Q}=\text { Delivery (in } \mathrm{l} / \mathrm{s}) ; \\
\mathrm{N}=\text { Power absorbed (in kW) }
\end{gathered}
$$

\begin{tabular}{|c|c|c|c|c|c|c|c|c|c|c|c|}
\hline $\mathrm{H}$ & 4 & 4 & 4 & 4 & 4 & 4 & 4 & 4 & 4 & 4 \\
\hline $\mathrm{Q}$ & 550( & 425 & 340 & 280 & 225 & 112 & 85 & 50 & 23 & 0 \\
\hline $\mathrm{N}$ & 35 & 21.5 & 17 & 14 & 12.2 & 10.5 & 9.6 & 8.2 & 7 & 6.3 \\
\hline
\end{tabular}


(3) PUMP With VARIABLE-PITCH BLADES :

(blade pitch angle varying from $21^{\circ}$ to $6^{\circ}$ ) and characterized by the following figures, at constant speed :

$\mathrm{H}=$ Lift (in $\mathrm{m}$ ); $\mathrm{Q}=\operatorname{Delivery~(in~} \mathrm{l} / \mathrm{s}$ ); $\mathrm{N}=$ Power absorbed (in kW)

\begin{tabular}{|c|c|c|c|c|c|c|c|c|c|}
\hline $\mathrm{H}$ & 2 & 3 & 4 & 5 & 6 & 7 & 9 & 11 & 14 \\
\hline $\mathrm{Q}$ & 390 & 370 & 340 & 315 & 275 & 150 & 70 & 45 & 0 \\
\hline $\mathrm{N}$ & 12.5 & 14 & 17 & 20 & $22.5 \mid$ & 18 & 19 & 19.5 & 20 \\
\hline $\mathrm{B}$ & $21^{\circ}$ & $21^{\circ}$ & $21^{\circ}$ & $21^{\circ}$ & $21^{\circ}$ & $15^{\circ}$ & $12^{\circ}$ & $9^{\circ}$ & $6^{\circ}$ \\
\hline
\end{tabular}

Or, at constant lift :

$$
\begin{gathered}
\mathrm{H}=\text { Lift (in } \mathrm{m} \text { ); } \quad Q=\text { Delivery (in } 1 / \mathrm{s}) ; \\
\mathrm{N}=\text { Power absorbed (in } \mathrm{kW})
\end{gathered}
$$

\begin{tabular}{|c|c|c|c|c|c|c|c|c|c|}
\hline $\mathrm{H}$ & 4 & 4 & 4 & 4 & 4 & 4 & 4 & 4 & 4 \\
\hline $\mathrm{Q}$ & 550 & 425 & 340 & 280 & 225 & 114 & 47 & 27 & 0 \\
\hline $\mathrm{N}$ & 35 & 21.5 & 17 & 14 & 12.2 & 7.8 & 5.6 & 4.3 & 3.1 \\
\hline
\end{tabular}

After determining for each type of pump the power-speed curves, we obtain the delivery-wind speed curves. The shape of these curves near cutin is very different according to whether the pump is a propeller type or a centrifugal. The calculation of the total volume pumped in the year and of the number of hours of operation gives the following results, still for a rated power established at $8 \mathrm{~m} / \mathrm{s}$.

\section{Centrifugal pump :}

Cut-in speed $v=6,75 \mathrm{~m} / \mathrm{s}$.

Annual volume of water $\mathrm{V}=4,686,000 \mathrm{~m}^{3}$.

Number of hours of operation $=4,190$.

Propeller pump :

Cut-in speed $v=0 \mathrm{~m} / \mathrm{s}$.

Annual volume of water $\mathrm{V}=4,953,000 \mathrm{~m}^{3}$.

Number of hours of operation $=5,250$.

Pump with variable - pitch blades :

Cut-in speed $v=4.5 \mathrm{~m} / \mathrm{s}$.

Annual volume of water $\mathrm{V}=5,455,500 \mathrm{~m}^{3}$.

Number of hours of operation $=6,850$.

The propeller pump with fixed blades enables the volume pumped to be increased by $6 \%$ and the time of operation by $25 \%$. The pump with variable-pitch blades increases the volume pumped by $17 \%$ and the time of operation by $63 \%$ in comparison with the centrifugal pump under the same conditions.

These figures show clearly the advantage of using a propeller pump and especially a pump with variable-pitch blades. The latter certainly necessitates greater mechanical complication, but, for machines of a certain power, the gain in volume of water pumped and especially in length of time of operation is such that this complication is completely justified. Although the use of the pump with variable-pitch blades remains practically limited to lifts of the order of 6 to $8 \mathrm{~m}$ at the maximum, on the ofher hand, the propeller pump may be used for much grealer lifts in an arrangemenl with several stages in series.

\section{CONCLUSION}

There are, at present, some types of windmills which are perfectly suitable for driving pumps, either the slow-running windmill for a piston pump, or the high-speed windmill for a centrifugal or propeller pump. All of them, in their particular field, give a large hydraulic power output and a satisfactory number of hours of operation. For modera. tely windy districts, these sets are quite economic and can compete very successfully with electrica? and thermal pumping sets. Their superiority is obvious for use in remote places or where there is no distribution network. If there have been failures, they must be attributed more to lack of information on the winds or to the use of unsuitable equipment than to a fault inherent in pumping by wind energy. In all cases, and especially for high-speed sets of a certain size, the installation must be based on a preliminary survey of the wind regime and on a serious study of the suitability of the characteristics of the machines used. When these conditions are fulfilled, the windmill will provide an economic solution to many pumping problems. 